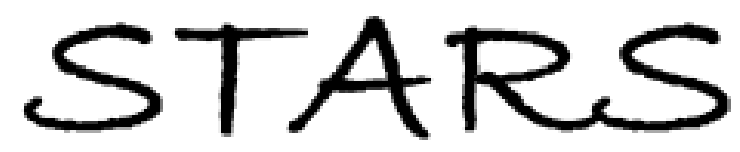

University of Central Florida

STARS

$1-1-2008$

\title{
Mode selection and phase locking of sidelobe-emitting semiconductor laser arrays via reflection coupling from an external narrow-bandwidth grating
}

\author{
S. Riyopoulos \\ G. Venus \\ University of Central Florida \\ L. Glebov \\ University of Central Florida
}

Find similar works at: https://stars.library.ucf.edu/facultybib2000

University of Central Florida Libraries http://library.ucf.edu

This Article is brought to you for free and open access by the Faculty Bibliography at STARS. It has been accepted for inclusion in Faculty Bibliography 2000s by an authorized administrator of STARS. For more information, please contactSTARS@ucf.edu.

\section{Recommended Citation}

Riyopoulos, S.; Venus, G.; and Glebov, L., "Mode selection and phase locking of sidelobe-emitting semiconductor laser arrays via reflection coupling from an external narrow-bandwidth grating" (2008).

Faculty Bibliography 2000s. 892.

https://stars.library.ucf.edu/facultybib2000/892

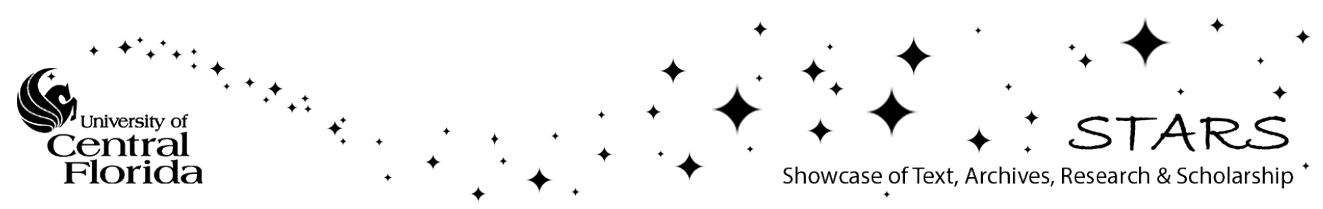




\section{Mode selection and phase locking of sidelobe-emitting semiconductor laser arrays via reflection coupling from an external narrow-bandwidth grating}

Cite as: J. Appl. Phys. 103, 113107 (2008); https://doi.org/10.1063/1.2936970

Submitted: 03 October 2007 . Accepted: 29 March 2008 . Published Online: 09 June 2008

S. Riyopoulos, G. Venus, and L. Glebov

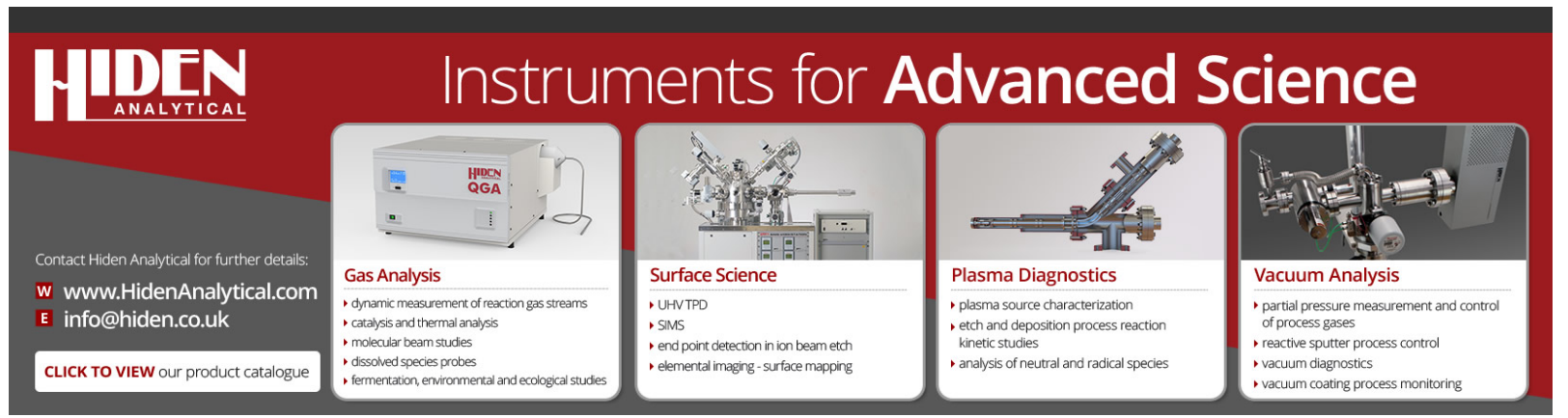




\title{
Mode selection and phase locking of sidelobe-emitting semiconductor laser arrays via reflection coupling from an external narrow-bandwidth grating
}

\author{
S. Riyopoulos, ${ }^{1, a)}$ G. Venus, ${ }^{2}$ and L. Glebov ${ }^{2}$ \\ ${ }^{1}$ Science Applications International Corporation, McLean, Virginia 22102, USA \\ ${ }^{2}$ CREOL, University of Central Florida, Orlando, Florida 32816, USA
}

(Received 3 October 2007; accepted 29 March 2008; published online 9 June 2008)

\begin{abstract}
A phase locked array design, utilizing direct reflection feedback between adjacent cavities by an external grating, is analyzed and proposed. The narrow grating reflection bandwidth causes longitudinal mode selection, while the array geometry causes transverse wavenumber selection through the coupling strength. As a result, only one among the free running cavity eigenmodes can couple effectively into a phase locked collective eigenmode. The coupled array mode is experiencing the high reflectivity of the grating and surpasses the low gain of the free running modes, that experience only a much lower reflectivity from the cavity edge antireflective coating. These results suggest that in-phase locking and single mode operation can be achieved simultaneously through the use of an external narrow-bandwidth grating. () 2008 American Institute of Physics. [DOI: 10.1063/1.2936970]
\end{abstract}

\section{INTRODUCTION}

Coherent phase locking of semiconductor laser arrays ${ }^{1-3}$ is attractive for high power applications ranging from material processing to free space communications to coherent beam combining. Single mode operation of each laser cavity is necessary for achieving phase locking and phase control. Although several coupled array approaches have been proposed so far, including evanescent coupling, antiguided (photonic mode) arrays, ${ }^{4}$ and external feedback synchronization, ${ }^{5}$ maintaining phase coherence and single mode a high power levels remains a challenge.

The recent advent of Bragg gratings recorded in photothermorefractive (PTR) glass allows extremely small reflection bandwidth $\delta K_{R}$, smaller than the longitudinal mode separation in the cavity $\Delta k_{z}=\pi / L$, through a combination of very low index contrast $\delta n / n \sim 10^{-4}$ (for small $\delta K_{R}$ ) with a large number of quarter-wavelength pairs $\sim 10^{3}-10^{4}$ (for high reflectivity) and extremely low absorption. That enables a new approach to phase locking based on reflection coupling between sidelobe emitting broad area lasers inside a shared external cavity. The first experimental demonstration of single-mode phase locking of two multimode laser diodes was described in Ref. 6. However, the feasibility of this approach for multicavity laser arrays is not obvious and, therefore, is the goal of this work.

The concept is schematically illustrated in Fig. 1. Broad area multistripe lasers emit far-field patterns forming sidelobes at angles $\theta=\tan ^{-1}\left(k_{x} / k_{z}\right)$, where $k_{x}$ corresponds to some transverse mode wavenumber. The output of each cavity is Bragg reflected by the external grating directly into the neighboring cavities. The low reflectivity from the antireflection coating (AR) at each cavity output causes very high lasing threshold for free running (uncoupled by the grating)

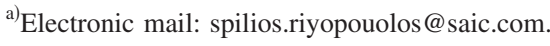

modes. Thus, the uncoupled free running modes remain below lasing threshold for usual operating currents. Only the grating-coupled collective array modes, that receive much higher reflectivity from the Bragg mirror, are raised above threshold for the same current. Since the narrow PTR bandwidth favors only one longitudinal value $k_{z}$ for reflection, and since the reflection geometry selects one sidelobe angle $\theta$ for coupling, meaning a single transverse wavenumber $k_{x}$ $=k_{o} \sin \theta$, only one among the free-running cavity modes $\omega\left(k_{z}, k_{x}\right)$ become coupled and thus raised above the lasing threshold. In other words, the proposed arrangement aims at concurrent achievement of phase locking and single mode control. Suppression of the free running vertical mode $\theta=0$, which stands out in gain due to direct reflection from the PTR, can be achieved either via selection of the material gain regime so that $g\left[\omega\left(k_{z}, k_{x}=0\right)\right]<0<g\left[\omega\left(k_{z}, k_{x} \neq 0\right)\right]$, or by tailoring a vertical antiresonant interference between the AR and the PTR.

Given the narrow bandwidth, thermal wavenumber drifts due to grating material expansion can be important for operation. The achieved low PTR glass absorption coefficient

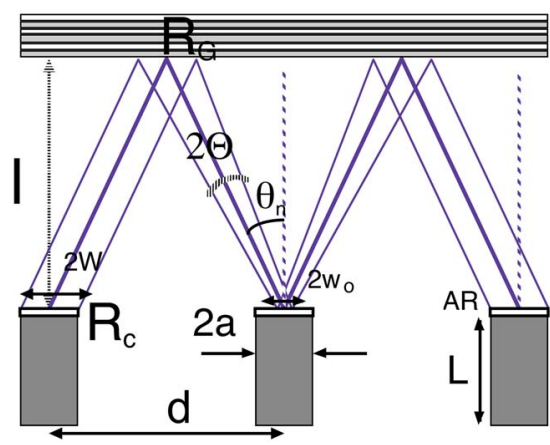

FIG. 1. (Color online) Schematic illustration of a sidelobe-emitting semiconductor laser array coupled via direct reflection from a narrow bandwidth external grating. 
$\simeq 10^{-3} \mathrm{~cm}^{-1}$, however, minimizes such effects. Computations yield a total expansion of $0.5 \mu \mathrm{m}$ for a typical grating thicknesses $2.5-3.0 \mathrm{~mm}$, corresponding to 7000-9000 grating periods for $n=1.5$ and a free space wavelength $\simeq 1 \mu \mathrm{m}$. The shift in the grating period and the reflection band center is then of the order $10^{-4} \mu \mathrm{m}$, too small to be of practical importance. A spatially nonuniform expansion of the total grating thickness, in response to a Gaussian beam power distribution, could be more significant than a uniform frequency shift, yet the corresponding lensing effects are still relatively small.

The rest of this paper is organized as follows. The general coupled-cavity rate equations for an edge emitting semiconductor laser array are obtained and discussed in Sec. II. The coupling coefficients for sidelobe emitting broad-stripe lasers are derived in detail in Sec. III due to their importance in determining the complex coupling strength among cavities. The distributed reflection from a thick, low-indexcontrast grating is treated in detail in Sec. IV in order to determine the effective reflection and diffraction lengths which are longer than the grating-cavity separation. The derivation of the feedback-coupled collective array modes and the discussion of the threshold gain and mode control, the main result of this work, appear in Sec. V. Vertical mode control is discussed in Sec. VI.

\section{COUPLED-CAVITY RATE EQUATIONS}

We assume single polarization cavity modes with profiles of the form

$$
\mathbf{E}=\mathcal{E}(z, t) U(x, y) e^{i k_{x} x+i k_{z} z-i \omega t} \hat{\mathbf{y}},
$$

where $k_{x}, k_{z}$ carry the "fast phase" variation and $U^{n p}(\mathbf{r})$ with $\mathbf{r}=(x, y)$ is the cold-cavity (zero gain) eigenmode profile, solution of

$$
\nabla^{2} U^{n p}(\mathbf{r})+\left[\Delta \epsilon(\mathbf{r}) \frac{\omega^{2}}{c^{2}}+\epsilon_{o} \kappa_{n p}^{2}\right] U^{n p}(\mathbf{r})=0 .
$$

Here $\kappa_{n p}^{2} \equiv \omega^{2} / c^{2}-k_{n}^{2}-k_{p}^{2}$ carries the wavenumber quantization $\mathbf{k}=\left(k_{x}, k_{z}\right)=(n \pi / a, p \pi / L)$ for a slab waveguide of length $L$ with complex dielectric $\epsilon_{o}$ extending between $|x|$ $\leq a,|y| \leq h,|z| \leq L$ and $\Delta \epsilon(\mathbf{r})$ signifies index profile modification to thermal effects. The evolution of the slowly varying envelope $\mathcal{E}(z, t)$ under the complex gain influence is given by

$$
\left(\frac{\partial}{\partial t}+v_{g} \frac{\partial}{\partial z}\right) \mathcal{E}=-i 4 \pi \frac{\chi(\omega)}{\frac{\partial(\omega \epsilon)}{\partial \omega}} \mathcal{E} \equiv g(\omega) \mathcal{E},
$$

where $\chi$ is the complex material susceptibility, the complex material gain is defined by $g=g^{\prime}+i g^{\prime \prime} \equiv-i 4 \pi \chi(\omega)$ $\times[\partial(\omega \epsilon) / \partial \omega]^{-1}$, the group velocity is given by $v_{g}=d \omega / d k_{z}$, and $\partial(\omega \epsilon) / \partial \omega$ is the usual factor relating energy density to the amplitude in dispersive materials. For weak dispersion $d \epsilon / d \omega \ll 1$, one has $v_{g} \simeq v_{p}$ and $\partial(\omega \epsilon) / \partial \omega \simeq \epsilon$.

Without loss of generality we consider a $1 \times N$ array with cavity separation (pitch) $d>2 a$ along $x$. For the moment we assume identical cold cavity parameters, imposing invariance under lateral displacements by an array period $d$. Thus, the array gain and reflectivity profiles are given by
$\tilde{g}(\mathbf{r})=g_{\infty}+\sum_{j=0}^{N} g_{o} \zeta_{g}(\mathbf{r}-j d)$ and $\tilde{R}(\mathbf{r})=R_{\infty}+\sum_{j=0}^{N} R_{c} \zeta_{m}(\mathbf{r}-j d)$, where $g_{\infty}, R_{\infty}$ are constant "floor" values between the lasing cavity regions. To simplify the computation we assume flat profiles $\zeta_{g, m}(\mathbf{r}-j d)=1$ for $|x-j d|<a,|y|<h$ and $\zeta_{g, m}(\mathbf{r})=0$ otherwise, where the cavity half width $a<d / 2$. We also assume that cavity coupling involves identical mode numbers with identical profiles $U^{n p}=U$, barring accidental frequency degeneracy. Thus, we consider array mode envelopes given by a superposition of cavity eigenmode envelopes

$$
\widetilde{\mathcal{E}}=\sum_{j=0}^{N} \mathcal{E}_{j}(z, t) U(\mathbf{r}-j d)=\sum_{j=0}^{N} \mathcal{E}_{j}(z, t) U_{j}(\mathbf{r}),
$$

using the notation $U_{j}(\mathbf{r}) \equiv U(\mathbf{r}-j d)$. In subsequent calculations one can simply write, evoking periodicity, $U_{j \pm 1}(\mathbf{r}) U_{j}(\mathbf{r})=U_{1 \pm 1}(\mathbf{r}) U_{1}(\mathbf{r})=U(\mathbf{r} \mp \mathbf{d}) U(\mathbf{r})$, etc.

Substituting the collective envelope Eq. (4) inside Eq. (3), taking the projection with the $j$ th cavity profile $U_{j}(\mathbf{r})$, integrating over the array volume contained between the SL cavity edges, and applying Stokes theorem converting volume integrals into surface integrals, capturing the edge cavity emitted flux and the intercavity reflected flux, yield a set of coupled cavity envelope equations (Appendix A),

$$
\sum_{j=i-1}^{j=i+1} C_{i j} \frac{\partial \mathcal{E}_{j}}{\partial t}=v_{g}\left[g \Upsilon_{i j}-\mu M_{i j}+\nu V_{i j}\right] \mathcal{E}_{j} .
$$

The reflection loss and grating feedback constants are given via the power reflectance and transmittance as $\mu=T_{c} / 2 L$ $=\left(1-R_{c}\right) / 2 L=\left(1-|r|^{2}\right) / 2 L$, where $R$ is the reflectance and $r$ is the amplitude reflectivity. The factor $\mu / 2$ applies to the amplitude growth rate since $\partial E_{o} / \partial t=(1 / 2)\left(\partial E_{o}^{2} / \partial t\right)$. For high reflectance $R=1-\epsilon$ with $\epsilon \ll 1$, whereby $\epsilon \simeq-\ln R$, one has $\mu(1-R) / 2 L=\epsilon / 2 L$ recovering the familiar result $\mu \simeq$ $-\ln R / 2 L$. We adopt the exact $\nu, \mu$ definitions below Eq. (5) that are always valid and should be used for low $R<1$ values. Incidentally, the transmittance $T=1-|r|^{2} \neq|t|^{2}$ (Appen$\operatorname{dix} \mathrm{A})$, so the reflectivity $r$ is more convenient for expressing cavity losses.

The earlier general coupling equations contain both nearfield coupling terms, due to fringe field overlap, and far-field coupling due to reflection from the grating. The radiation overlap between neighboring cavity evanescent fields is $C_{i, i \pm 1}=\int d \mathbf{r}^{2} U_{i}(\mathbf{r}) U_{i \pm 1}(\mathbf{r})$. Only neighboring cavity coupling is considered here $j=i \pm 1$, though extension to arbitrary order coupling is straightforward. The mode profiles are normalized so that $C_{i i}=\int d \mathbf{r}^{2} U_{i}^{2}(\mathbf{r})=1$. The mode overlapping with the same-cavity gain profile (confinement factor) $g_{o} \zeta_{i}^{g}(\mathbf{r})$ and the overlapping with the edge mirror losses profile $\mu_{o} \zeta_{j}^{m}(\mathbf{r})$, given, respectively, by

$$
\begin{aligned}
& \Upsilon_{i i}=\int d \mathbf{r}^{2} U_{i}^{*}(\mathbf{r}) \zeta_{i}^{g}(\mathbf{r}) U_{i}(\mathbf{r}), \\
& M_{i i}=\int d \mathbf{r}^{2} U_{i}^{*}(\mathbf{r}) \zeta_{i}^{m}(\mathbf{r}) U_{i}(\mathbf{r}),
\end{aligned}
$$

are referred to as the gain and mirror "confinement factors." The corresponding cross-cavity near-field gain coupling and near-field reflection coupling, due to fringe-field overlap, are 


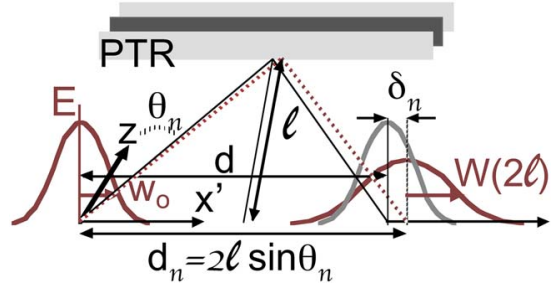

FIG. 2. (Color online) Schematic illustration of the overlapping between a cavity mode Gaussian envelope and the reflected off the grating, expanded (diffracted) sidelobe emanating from the cavity on the left. The offset between the center of the reflected sidelobe and the target cavity center is $\delta_{n}$ $=d-2 l \sin \theta_{n}$, with $d$ as the array pitch and $\theta_{n}$ as the sidelobe angle. The target and source cavity coordinates, respectively, $x, x^{\prime}$, are related by $x^{\prime}$ $=x+d$.

$$
\begin{aligned}
& Y_{i, i \pm 1}=\int d \mathbf{r}^{2} U_{i}^{*}(\mathbf{r}) \zeta_{i}^{g}(\mathbf{r}) U_{i \pm 1}(\mathbf{r}), \\
& M_{i, i \pm 1}=\int d \mathbf{r}^{2} U_{i}^{*}(\mathbf{r}) \zeta_{i}^{m}(\mathbf{r}) U_{i \pm 1}(\mathbf{r}) .
\end{aligned}
$$

Finally, the far-field reflection overlap between the expanded (diffracted) mode wavefront from cavity $i \pm 1$ and the $i$ th cavity wavefront, Fig. 2, is given by

$$
\begin{aligned}
V_{i, i \pm 1} & =\int d \mathbf{r}^{2} U_{i}^{*}(\mathbf{r} ; z=0) U_{i \pm 1}(\mathbf{r} ; z=2 l) \\
& =\int d \mathbf{r}^{2} U^{*}(\mathbf{r} ; 0) \mathcal{P}_{z=2 l} U(\mathbf{r}+d ; 0),
\end{aligned}
$$

where $\mathcal{P}$ is the paraxial propagator along $z$ distance twice the grating separation $l$. Due to near-neighbor coupling the coefficient matrices $\mathcal{C}=\left\{C_{i j}\right\}, \mathcal{Y}=\left\{\Upsilon_{i j}\right\}, \mathcal{M}$ $=\left\{M_{i j}\right\}, \quad \mathcal{V}=\left\{V_{i j}\right\} \quad$ are tridiagonal. Furthermore, array periodicity impose $Y_{i i}=\int d \mathbf{r}^{2} U_{i}^{*}(\mathbf{r}) \zeta_{i}^{g}(\mathbf{r}) U_{i}(\mathbf{r})$ $=\int d \mathbf{r}^{2} U^{*}(\mathbf{r}-i d) \zeta^{g}(\mathbf{r}-i d) U(\mathbf{r}-i d)=\int d \mathbf{r}^{2} U^{*}(\mathbf{r}) \zeta^{g}(\mathbf{r}) U(\mathbf{r})=\Upsilon_{11}$, and $\quad Y_{i, i \pm 1}=\int d \mathbf{r}^{2} U_{i}^{*}(\mathbf{r}) \zeta_{i}^{g}(\mathbf{r}) U_{i \pm 1}(\mathbf{r})=\int d \mathbf{r}^{2} U^{*}(\mathbf{r}-i d) \zeta^{g}(\mathbf{r}$ $-i d) U[\mathbf{r}-(i \pm 1) d]=\int d \mathbf{r}^{2} U^{*}(\mathbf{r}) \zeta^{g}(\mathbf{r}) U(\mathbf{r} \pm d)=\Upsilon_{12}=\Upsilon_{21}$, etc. hence, all diagonal elements are equal, and symmetric offdiagonal elements are also equal,

$$
\begin{aligned}
& \mathcal{Y}=\left(\begin{array}{lll}
\Upsilon_{11} & \Upsilon_{12} & \cdots \\
\Upsilon_{12} & \Upsilon_{11} & \Upsilon_{12} \\
\cdots & \Upsilon_{12} & \Upsilon_{11}
\end{array}\right), \\
& \mathcal{M}=\left(\begin{array}{lll}
M_{11} & M_{12} & \cdots \\
M_{12} & M_{11} & M_{12} \\
\cdots & M_{12} & M_{11}
\end{array}\right), \\
& \mathcal{V}=\left(\begin{array}{ccc}
0 & V_{12} & \cdots \\
V_{12} & 0 & V_{12} \\
\cdots & V_{12} & 0
\end{array}\right) .
\end{aligned}
$$

In matrix form

$$
\mathcal{C} \frac{\partial}{\partial t} \mathcal{E}=v_{g}[g \mathcal{Y}-\mu \mathcal{M}+\nu \mathcal{V}] \mathcal{E}
$$

The presence of nondiagonal coupling terms on the left-hand side time derivatives has the following meaning: any gain or loss from the right-hand side (rhs) of Eq. (10) is shared among neighbor cavities in proportion to the fringe field overlapping (since cavity eigenmodes are not "lattice orthogonal"). Multiplying both sides by $\mathcal{C}^{-1}$ diagonalizes the left-hand side, yielding the final form

$$
\frac{\partial}{\partial t} \mathcal{E}=v_{g}[g \hat{\mathcal{Y}}-\mu \hat{\mathcal{M}}+\nu \hat{\mathcal{V}}] \mathcal{E}
$$

where $\hat{\mathcal{Y}}=\mathcal{C}^{-1} \mathcal{Y}, \hat{\mathcal{M}}=\mathcal{C}^{-1} \mathcal{M}$, and $\hat{\mathcal{Y}}=\mathcal{C}^{-1} \mathcal{Y}$. In fact, expression (11) can be arrived at from the beginning by introducing a properly constructed lattice-orthogonal basis ${ }^{7,8} \hat{U}(\mathbf{r})$ satisfying $\int d \mathbf{r}^{2} \hat{U}_{i}(\mathbf{r}) \hat{U}_{j}(\mathbf{r})=\delta_{i j}$, in place of the original $U_{j}(\mathbf{r})$.

\section{SIDELOBE COUPLING}

The effect of near-field evanescent coupling has been extensively studied in Refs. 7 and 8 in association with closely packed vertical-cavity surface-emitting lasers arrays. In the considered sidelobe coupling scheme between edge emitters, Fig. 1, the cavity pitch $d$ is much larger than the mode waist $w_{o}$ and the near-field overlap is exponentially small and negligible compared to the far-field reflection coupling $\sim R_{G}$. Off-diagonal terms can be dropped from $\mathcal{Y}, \mathcal{M}$, and $\mathcal{C}$ becomes the identity matrix $\mathcal{I}$, whereby both Eqs. (10) and (11) are reduced to

$$
\frac{\partial}{\partial t} \mathcal{E}=v_{g}\left[g \Upsilon_{11} \mathcal{I}-\mu M_{11} \mathcal{I}+\nu \mathcal{V}\right] \mathcal{E}
$$

where the diagonal elements $\Upsilon_{11}, M_{11}$ are the cavity confinement factors. The only intercavity coupling comes from the (by definition off-diagonal) grating reflection matrix elements $\mathcal{V}$. Next we proceed in evaluating the elements $V_{j, j \pm 1}=V_{12}$ defined by Eq. (8).

Using Cosine-Gauss-Hermite products as the most general mode profiles for a stripe laser, following a rational similar to Refs. 2 and 3, the near field at the output $z=0$ is written as

$$
\begin{aligned}
& U_{m n p}(x, y)=s_{n p}(y)\left[u_{m n}^{+}(x)+u_{m n}^{-}(x)\right], \\
& s_{n p}(y)=B \cos \left(k_{y} y\right),|y|<h, \\
& s_{n p}(y)=B^{\prime} \exp \left(-k_{y}^{\prime} y\right),|y|>h, \\
& u_{m n}^{ \pm}(x)=C_{m n}\left(\frac{2}{\pi w_{o}^{2}}\right)^{1 / 4} H_{m}\left(\frac{\sqrt{2} x}{w_{o}}\right) e^{-x^{2} / w_{o}^{2}} e^{ \pm i k_{n} x} .
\end{aligned}
$$

Above $k_{x} \equiv k_{n}=n \pi / a$ and $k_{y}=\sqrt{\epsilon_{o} \omega^{2} / c^{2}-k_{n}^{2}-k_{p}^{2}}$ are the transverse wavenumbers along the cavity width $2 a$ and cavity height $2 h$, respectively, satisfying a slab-type dispersion relation $\omega_{n p}\left(k_{n}, k_{p}\right)$, where $p$ labels the longitudinal wavenumber $k_{z}=k_{p}=p \pi / L$. The Gauss-Hermite function $H_{m}(\chi) \exp \left[-\chi^{2} / 2\right]$, with $\chi \equiv \sqrt{2} x / w_{o}$, where the Gaussian waist is given by $w_{o}=\sqrt{a \lambda / 2 \pi \sqrt{2}\left(\Delta \epsilon_{o} / \epsilon_{o}\right)^{1 / 4}}$ and includes 
the effect of a parabolic index profiling (thermal lensing), $\Delta \epsilon(x)=(1 / 2) \Delta \epsilon_{o} x^{2} / a^{2}$ in Eq. (2). This also modifies the final dispersion into $\epsilon_{o} \omega_{m n p}^{2} / c^{2}-k_{n}^{2}-k_{p}^{2}-k_{y}^{2}=\Delta \epsilon_{o}(2 m+1)$.

The use of a collimating mirror with matched radius of curvature and focal distance is expected to focus the radiation in the "fast diffracting" $y$ direction and minimize the $y$-diffraction effects. We thus focus on the effects of the free diffraction along the $x$ axis during the round trip to the grating and into the adjacent cavity. For small transverse wavenumbers $k_{\perp} / k_{o} \ll 1$, the far field is given by the convolution of the paraxial propagator with the output near field $u(x ; z)$ $=\int d x^{\prime} u\left(x^{\prime} ; z=0\right) \sqrt{i k_{o} / 2 \pi z} \exp \left\{i k_{o}\left[\left(x^{\prime}-x\right)^{2} / 2 z\right]\right\}$, where $k_{o}$ $=\omega / c$. Using the generating function expansion for the Hermite-Gaussian yields (Appendix B),

$$
\begin{aligned}
u_{m n}^{+}(x ; z)= & C_{m n}\left[\frac{2}{\pi W^{2}(z)}\right]^{1 / 4} e^{-i(m+1 / 2) \varphi(z)} H_{m} \\
& \times\left[\frac{\sqrt{2}\left(x-z \sin \theta_{n}\right)}{W(z)}\right] \\
& \times e^{-[1+i(z / b)]\left[\left(x-z \sin \theta_{n}\right)^{2} / W^{2}(z)\right]} e^{-i k_{o}\left(x^{2} / 2 z\right)},
\end{aligned}
$$

where $W^{2}(z)=w_{o}^{2}\left(1+z^{2} / b^{2}\right)$ with $b=k_{o} w_{o}^{2} / 2$ the diffraction length, $\varphi(z)=\tan ^{-1} z / b$, and $C_{m n}$ is a normalization factor. The far-field expression $u_{m n}^{-}(x ; z)$ follows by $\sin \theta_{n} \rightarrow$ $-\sin \theta_{n}$. Under the paraxial approximation $\theta_{n} \ll 1$, the argument $x \pm z \sin \theta_{n} \simeq x \cos \theta_{n} \pm z \sin \theta_{n}$ is the $x^{\prime}$ coordinate in a rotated frame with $z^{\prime}$ aligned with $\pm \theta_{n}$. Hence, a CosineGauss-Hermite mode diffracts as a pure Gauss-Hermite along a propagation axis rotated by $\theta_{n}$. For the fundamental Gaussian mode $m=0$, the radiation peaks at $x \pm z \sin \theta_{n}=0$ forming two symmetric sidelobes at angles $\pm \theta_{n}$ $\equiv \sin ^{-1}\left( \pm k_{x} / k_{o}\right)=\sin ^{-1}\left( \pm k_{n} / k_{o}\right)$ relative to the $z$ direction with $k_{o}=\omega / c$ as the vacuum wavenumber. The angular divergence of each sidelobe around $\theta_{n}$, defined at the $1 / e$ folding angle, is given by $\Theta \simeq W(z) / z \rightarrow w_{o} / b=\lambda_{o} / \pi w_{o}$.

The coupling coefficient due to the reflected far-field incidence onto the adjacent cavity near field is written as $R_{12} V_{12}$, where $R_{12}=T_{c} R_{G} T_{c}$ is the product of the grating reflection coefficient $R_{G}$ times two transmissions through the AR coatings with reflection coefficient $R_{c}$. The factor $V_{12}$ gives the overlap of the diffracted far field envelope with the near field of the right adjacent cavity. Because the cavity field is a standing wave between $e^{i k_{x} x}$ and $e^{-i k_{x} x}$ the right sidelobe overlap with the target cavity has two terms

$$
V_{12}=V_{m n}^{++}+V_{m n}^{+-} .
$$

Taking into account the cavity center separation $V_{m n}^{ \pm \pm}$ $\equiv \int d \mathbf{r}^{2} u_{m n}^{ \pm *}(x ; z=0) u_{m n}^{ \pm}(x+d ; z=2 l)$. The left sidelobe projection onto the left adjacent cavity follows directly from $V_{m n}^{-}$ $=\left\{V_{m n}^{++}\right\}^{*}, V_{m n}^{++}=\left\{V_{m n}^{+-}\right\}^{*}$. The values $V_{0 n}^{ \pm \pm}, V_{0 n}^{ \pm \mp}$ for the fundamental Cosine-Gaussian $m=0$ are computed in Appendix C. The interference $V^{+-}$between opposite $\pm k_{n}$ sidelobes is exponentially smaller by $e^{-4 b^{2} \sin ^{2} \theta_{n} / w_{0}^{2}(1+\xi) \ll 1}$ and can be neglected. The sidelobe coupling strength $\left(1-R_{c}^{2}\right) R_{G} V_{12}$ enters the coupled cavity Eqs. (12) in the form $\nu V_{12}^{\prime}$ where $\nu \equiv(1$ $\left.-R_{c}^{2}\right)\left|R_{G}\right| / 2 L$ and the reflection phase shift $\chi$ at the grating is absorbed inside $V_{12}^{\prime} \simeq(1 / 4) V^{++} e^{i \chi}$,

$$
\begin{aligned}
V_{12}= & \frac{1}{2}\left[\frac{4}{4+(2 l / b)^{2}}\right]^{1 / 4} \\
& \times \exp \left\{-\frac{2 \delta^{2}}{w_{o}^{2}\left[4+(2 l / b)^{2}\right]}\right\} \exp (i\{\chi-\varphi(2 l) \\
& \left.\left.+\frac{1}{2} \varphi(l)+\frac{k_{o} X_{n}\left(X_{n}+2 \delta\right)}{2(2 l)}-\frac{(2 l / b) \delta^{2}}{w_{o}^{2}\left[4+(2 l / b)^{2}\right]}\right\}\right) .
\end{aligned}
$$

For on-center incidence $\delta=0$, the coupling scales as $\{4 /[4$ $\left.\left.\left.+(2 l / b)^{2}\right]\right\}\right\}^{1 / 4}$. For reflection path lengths shorter than the diffraction length $2 l<b$, the expansion in the reflected mode spot size is small and the overlap factor near $50 \%$ (half the power in each sidelobe). For significant coupling the diffraction spread in the far field cannot be large, requiring round trip lengths $2 l \leq b$. This is why the full paraxial (Fresnel) propagator was employed instead of the far-field (Fraunhofer) limit $l / b \gg 1$.

Due to the distributed, multilayer interaction with the grating an effective distance $\hat{l}$ must replace the nominal distance $l$ (the distance of the leading grating edge) inside the coupling formulae. It is shown next that separate effective distances apply for finding the beam path and the beam spotsize expansion.

\section{EFFECTIVE GRATING DISTANCE DUE TO DISTRIBUTED REFLECTION}

Distributed reflection over the long penetration depth inside the thick, low-index contrast, Bragg grating causes significant changes in the reflected wavefront. The total reflected radiation at the leading grating edge $z=l$ is a superposition of partial wavefronts $d E(z)$ reflected from different depths $\bar{z} \equiv z-l$ within the grating thickness $G$. Due to different traveled distances $2 \bar{z}$, wavefronts reflected off deeper layers have increasingly expanded (diffracted) $1 / e$ widths $W(l+\bar{z})$, and laterally displaced centers by $x$ $=2 \bar{z} \sin \theta$, as shown in Fig. 3(a). The strength of each reflected wavefront equals the reflected fraction $d E(\bar{z})$ from the depth $\bar{z}$. For gratings with low index contrast the coupled wave theory offers a very good approximation for the intensity inside the grating $d E(\bar{z})=d \bar{z} \kappa E_{o} e^{-\kappa \bar{z}}$, where $\kappa$ is related to the reflectance $R$ by $\kappa=-\ln R / 2 G$ and $G$ is the grating length. The superposition of partially reflected wavefronts, diffracted along the optical paths $2 \bar{z} \sin \theta^{\prime}$, yields the reflected wavefront at the leading edge $z=l$ (Appendix D),

$$
\begin{aligned}
E^{\prime}(x, y ; l)= & \sqrt{\frac{n^{2} k b}{\pi}} \int_{0}^{G} d \bar{z} \kappa \\
& \times \exp [-\kappa \bar{z}] \frac{1}{(n l+\bar{z})+i n b} \\
& \times \exp \left\{i n k \frac{\left(x-2 \bar{z} \sin \theta_{x} / n\right)^{2}+\left(y-2 \bar{z} \sin \theta_{y} / n\right)^{2}}{2[(n l+\bar{z})+i n b]}\right\}
\end{aligned}
$$

(generalized for oblique incidence in both $x$ and $y$ ). Here $\sin \theta^{\prime}=\theta / n$ is the diffraction angle inside the grating of 
(a)

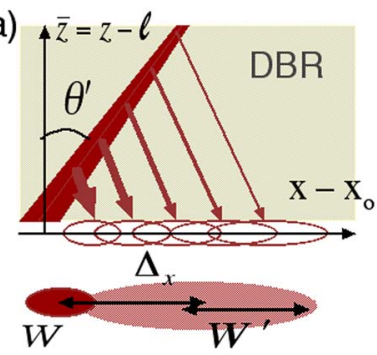

(b)
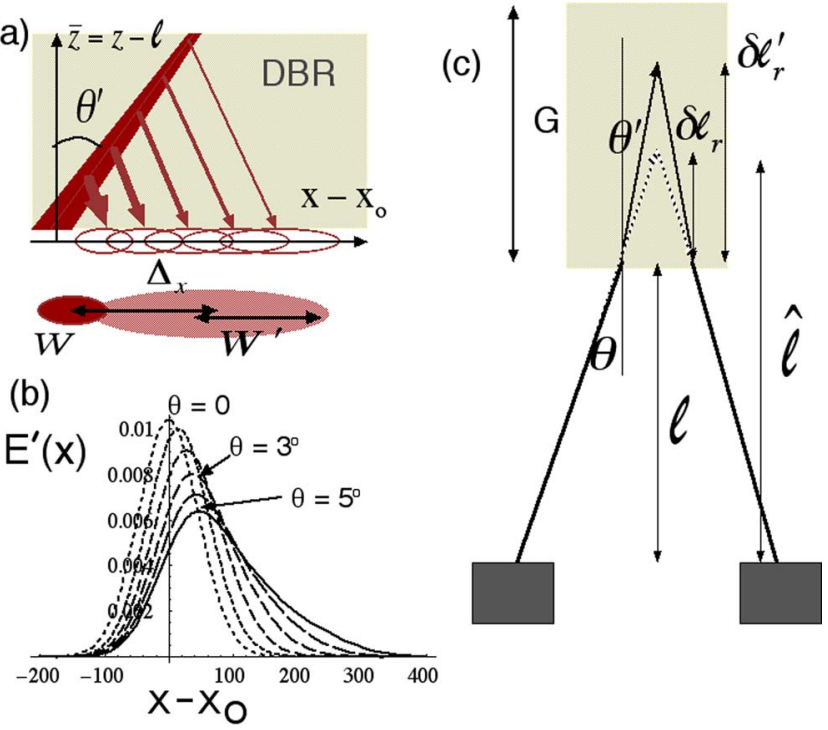

FIG. 3. (Color online) (a) Schematic illustration of distributed reflection through a DBR, showing the partial reflected wavefronts arriving back at the leading edge $z=l$. (b) The reflected field profile $E^{\prime}(x)$ for various incidence angles $\theta$, showing a shift in the peak location $\Delta_{x}$ and a spread in the spot size $W^{\prime}$. Incident beam waist $w_{o}=60 \mu \mathrm{m}$, grating thickness $G=2440 \mu \mathrm{m}$, distance $l=4000 \mu \mathrm{m}$, and reflection constant $\kappa=0.0011$ corresponding to reflectance $R=0.995$. (c) Relation between material reflection depth $\delta l_{r}^{\prime}$, free-space effective reflection depth $\delta l_{r}$ and effective grating distance $\hat{l}$.

average index $n=\left(n_{1} g_{1}+n_{2} g_{2}\right) / 2\left(g_{1}+g_{2}\right)$; the pair thicknesses $g_{1}, g_{2}$ are defined so that $k\left(n_{1} g_{1}+n_{2} g_{2}\right)=\pi$ at center bandwidth. Expression (18) is exact within the paraxial framework but can be computed only numerically. Figure 3(b) plots the reflected electric field profile, the real part of Eq. (18), for various incidence angles, by a grating of thickness $G \simeq 2440 \mu \mathrm{m}$ placed at distance $l=4000 \mu \mathrm{m}$. (For $\lambda_{o}$ $=0.980 \mu \mathrm{m}$ and average refraction index $\bar{n}=1.488 \mathrm{G}$ contains about 7400 quarter-wavelength pairs. A pair index contrast $\delta n=0.000433$ yields vertical reflectance of 0.9955 .) There is a lateral shift of the beam axis (peak) due to oblique incidence, and a beam spreading, caused by diffraction during propagation through the grating.

The lateral displacement in the beam axis $\Delta_{x}$ is calculated from the location of the maximum $\partial \Re E^{\prime} /\left.\partial x\right|_{x=\Delta_{x}}=0$, leading to an integral equation solved numerically (Appendix D). One can now define an effective penetration length $\delta l_{r}^{\prime}$ inside the grating via $\Delta_{x}=2 \delta l_{r}^{\prime} \sin \theta^{\prime}=2\left(\delta l_{r}^{\prime} / n\right) \sin \theta$, so that the axis shift from the distributed interaction equals to a shift from reflection by a mirror at depth $\bar{z}=\delta l_{r}^{\prime}$, corresponding to free-space depth $\delta l_{r}=\delta l_{r}^{\prime} / n$ [Fig. 3(c)]. The shift $\Delta_{x}$ and freespace penetration depth $\delta l_{r}$ are plotted versus the incidence angle $\theta$ for various values of the reflectance $\kappa$ in Figs. 4(a) and $4(\mathrm{~b})$, respectively. The curves $\delta l_{r}(\theta, \kappa)$ remain close to the energy penetration distance $\delta l_{e}=1 / 2 \kappa$. This is not surprising, since partial contributions are weighted by the reflected fraction and most power is reflected from within the energy penetration depth, Fig. 4(b). Hence, regarding the beam propagation path, one may treat reflection by the grating as reflection by a mirror located at an effective distance

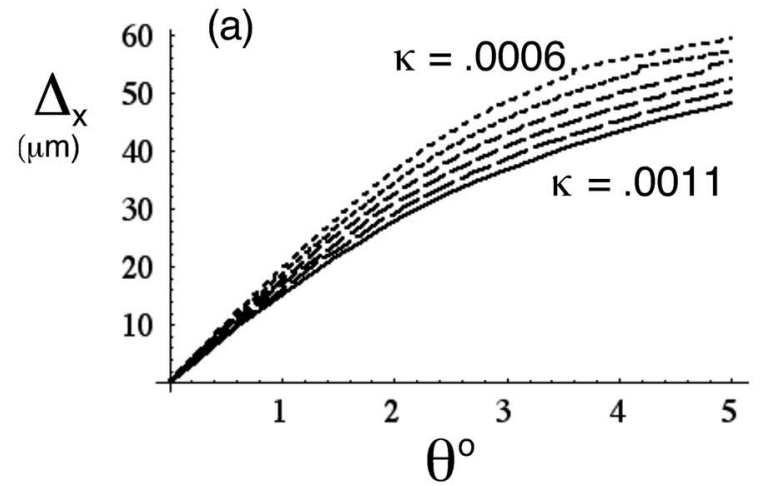

(b)
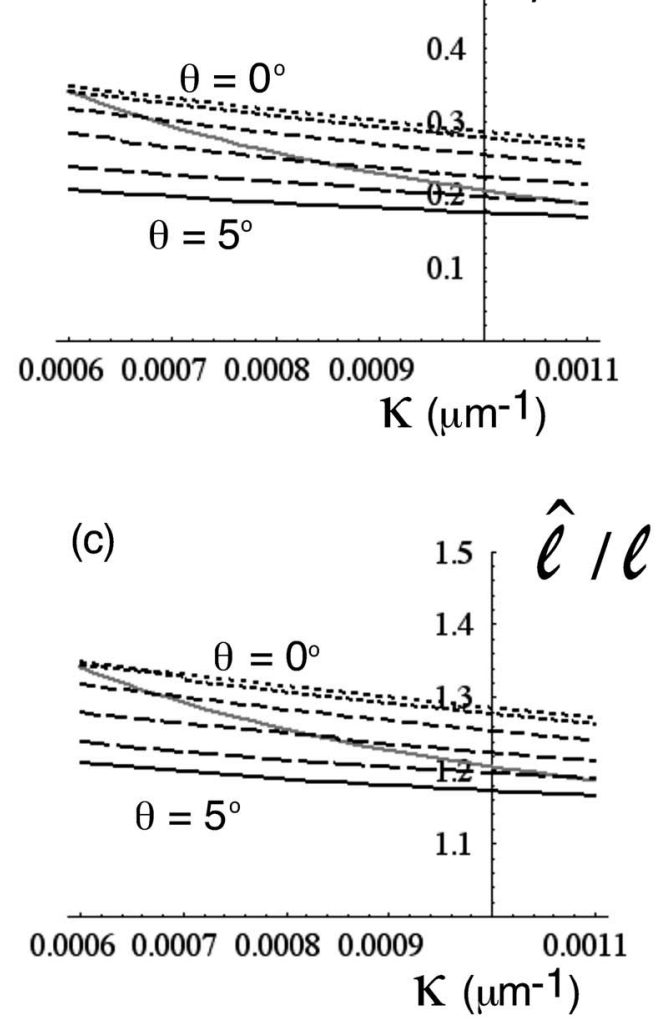

FIG. 4. Beam axis shift during distributed reflection from a grating of thickness $G=2440 \mu \mathrm{m}$. (a) Shift $\Delta_{x}$ of the reflected beam axis (at the grating's leading edge $z=l$ ) vs incidence angle $\theta$, for various reflection constants values as marked, and same other parameters as Fig. 3. (b) Ratio of effective penetration depth $\delta l_{r}$ to the grating thickness $G$ vs reflection constant $\kappa$, for various incidence angles $\theta$. Thick gray line marks the energy penetration depth $\delta l_{e} / G=1 / 2 \kappa G$. (c) Ratio of effective reflection distance to the grating edge distance $\hat{l} / l$. Thick gray line marks the relative energy penetration distance $\hat{l} / l=1+1 / 2 \kappa l$. For the chosen grating the plotted range of $\kappa$ yields reflectance values $R(\kappa)$ from 0.946 to 0.995 .

$\hat{l}=l+\delta l_{r} \simeq l+\delta l_{e}$. Plots of effective reflection-to-actual grating distance $\hat{l} / l$ are given in Fig. 4(c).

Analytic estimates of the expansion of the beam are possible by taking the mean value of the radiation spot size, since for near-Gaussian beams the 1/ $e$ width $W$ equals twice the root-mean-square (rms) spot size. Taking the spatial average around the shifted axis $\left\langle W_{x}^{2}\right\rangle=2\left\langle\left(x-\Delta_{x}\right)^{2}\right\rangle,\left\langle W_{y}^{2}\right\rangle=2\langle(y$ $\left.\left.-\Delta_{y}\right)^{2}\right\rangle$ over radiation power yields the spot size in each direction (Appendix D), 


$$
\begin{aligned}
\left\langle W_{x, y}^{2}\right\rangle= & \frac{1}{R} \int_{0}^{G} d \bar{z} 2 \kappa \exp [-2 \kappa \bar{z}]\left\{w_{o}^{2}\left[1+\frac{(n l+\bar{z})^{2}}{(n b)^{2}}\right]\right. \\
& \left.+8 \sin ^{2} \theta_{x, y}\left(\frac{\bar{z}^{2}}{n^{2}}-2 \delta l_{x, y} \frac{\bar{z}}{n}+\delta l_{x, y}^{2}\right)\right\} .
\end{aligned}
$$

The first contribution averages the expanded (diffracted) wavefronts reflected from different depths, weighted by the reflected power fraction at each depth, and is independent of the incidence angle. The second contribution comes from the oblique incidence $\theta_{x, y} \neq 0$ and constitutes a nondiffractive spot-size elongation ("stretching") due to the lateral displacement of the reflected wavefront centers (weighted by the reflected power fraction.) This oblique stretching exceeds the first term inside Eq. (19) even at small angles. For oblique incidence in only one direction (say $x$ ) the reflected Gaussian becomes elliptic with $W_{x}>W_{y}$.

An effective diffraction depth $\delta l_{d}$ can now be defined, so that the reflected spot size $\left\langle W^{2}\right\rangle$ at the grating's leading edge $l$ equals a free-diffracted Gaussian width after a total propagation distance $l+2 \delta l_{d}$, i.e., $\left\langle W^{2}\right\rangle=w_{o}^{2}\left\{1+\left[\left(l+2 \delta l_{d}\right)^{2} / b^{2}\right]\right\}$. Then the spot size reflected from a thick grating is equal to that reflected from a mirror (of same complex reflectivity) placed at the effective diffraction distance $\bar{l}=l+\delta l_{d}$. It then follows that $\bar{l}=\left[\left(l+2 \delta l_{d}\right)+l\right] / 2=\left[b \sqrt{\left\langle W^{2}\right\rangle / w_{o}^{2}-1}+l\right] / 2$. The difference $\delta l_{d} \equiv \bar{l}-l$ defines the penetration depth inside the grating for diffraction calculations. Again, because radiation decays exponentially inside the grating, most of the radiation is reflected from the front layers and, for large reflectance, the penetration depth is much smaller than the grating thickness $G$. Yet, since the grating thickness is large compared to the incoming waist, the stretching $\simeq 2 \delta l_{d} \sin \theta$ in the reflected waist can be significant. Figure 5(a) shows the ratio of reflected spot size to beam waist, $W^{\prime} / w_{o}$, versus the beam waist for various incidence angles. Figure 5(b) shows the corresponding ratio of the effective diffraction distance to the grating distance $\bar{l} / l$ for $\kappa=0.0011\left(R_{G}=0.995\right)$ and the same parameters as before. For inclined incidence, the diffraction distance can be a few times the grating separation $l$. The spot-size expansion is reduced with increasing beam waist, yet the effective diffraction distance increases with $w_{o}$. This happens because it takes a path correction of the order the diffraction length $b, \delta l_{d} \sim b \propto w_{o}^{2}>l$, to account for any significant expansion in waist. All that matters to the coupling strength is the waist expansion. For on-center beam incidence to the target cavity $\delta=0$, Eq. (17) yields $\left|V_{12}\right| \simeq[1$

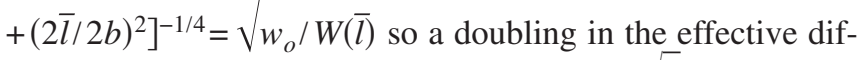
fraction length reduces coupling by about $1 / \sqrt{2}$.

It must be noted that the present calculation assumed a constant reflection coefficient over a Gaussian wavefront. Since the reflectivity of a grating depends on the axial wavenumber $k_{z}$, and since a Gaussian involves an axial spread $\Delta k_{z} / k \simeq 1 /\left(k w_{o}\right)^{2}$, constant reflectivity implies an axial Gaussian spectral width $\Delta k_{z}$ smaller than the grating reflection bandwidth $\sigma_{k}$. Because the latter must be smaller than the longitudinal mode separation $\Delta k_{z} / k=1 /(k L)$ for mode control, a large enough Gaussian waist is desired at the laser output so that
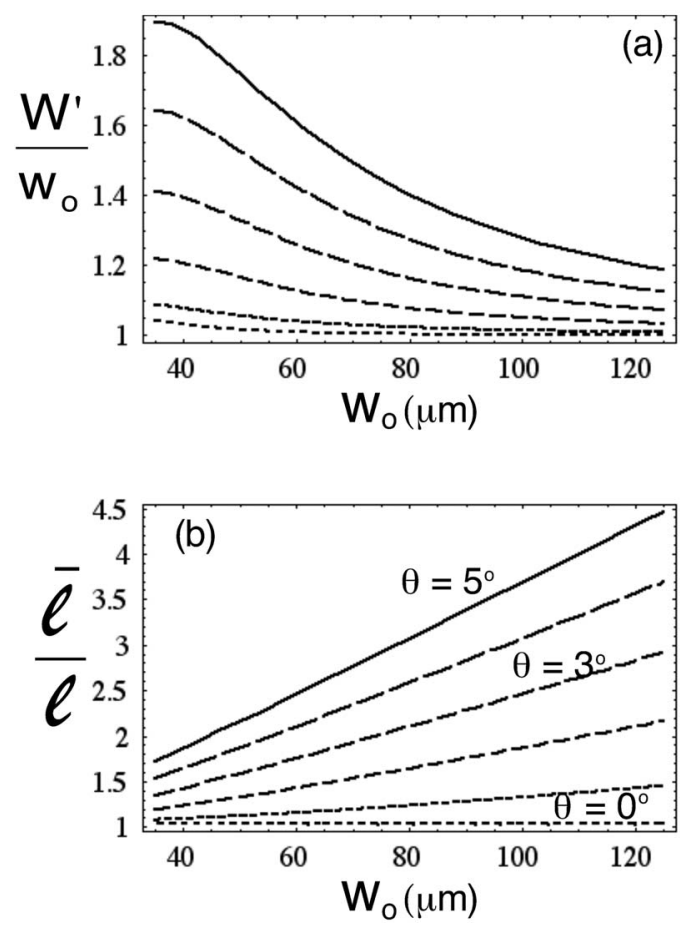

FIG. 5. Beam expansion during distributed reflection. (a) Ratio of the rms reflected spot size (at the grating's leading edge $z=l$ ) $W^{\prime}$ to the incident beam waist $w_{o}$, vs the waist $w_{o}$, for various incidence angles $\theta$. (b) Ratio of effective diffraction distance to the grating edge distance $\hat{l} / l$ vs the waist $w_{o}$ for various incidence angles $\theta$. Reflectance fixed at $R=0.995(\kappa=0.0011)$, with same other parameters as in Figs. 3 and 4.

$$
1 /\left(k w_{o}\right)^{2}<\sigma_{k} / k<1 /(k L)
$$

requiring $w_{o}^{2}>L \lambda / 2 \pi$. This is satisfied for the elected parameters. For spectral spreads exceeding the grating bandwidth the reflectivity is considerably reduced by spectral filtering.

In conclusion, an effective grating distance $\hat{l}=l+\delta l_{e}$ applies for determining the axial path of the reflected beam. The actual angle $\theta$ for on-center sidelobe coupling is $\sin \theta$ $=d / 2\left(l+\delta l_{e}\right)$ (for paraxial angles $\left.\sin \theta \simeq \tan \theta\right)$. A different effective diffraction distance $\bar{l}=l+\delta l_{d}$ applies for the beam spot-size expansion, entering the overlap coupling formula (17). Optimum lateral coupling requires that the center of the reflected sidelobe fall on the center of the next cavity $d$ $-X_{n}=d-2 \hat{l} \sin \theta_{n} \equiv \delta_{n} \ll w_{o}$, corresponding to sidelobe angle $\tan \theta_{n} \equiv k_{n} / k_{z} \simeq d / 2 \hat{l}$. Thus, $\hat{l}$ determines the sidelobe center offset and the transverse mode number for optimum coupling $n_{o} \simeq k_{z} a d / 2 \pi \hat{l}=a \lambda_{o}$. The actual grating separation $l$ is found from the relation between $l, \hat{l}$, and $\theta$. Finally, the diffraction length $\bar{l}$ is determined from $l$ and $\theta$ via Eq. (19). The diffraction length $\bar{l}$ enters explicitly in the coupling coefficient Eq. (17), while $\hat{l}$ enters implicitly through the center off-set $\delta$.

The computed coupling coefficients and effective lengths are used to show that the coupling strength dependence on the array geometry can lead to transverse mode selection. Figure 5 shows the calculated magnitude $\left|V_{12}\right|$ versus cavity mode waist $w_{o}$ for typical cavity parameters $d$ $=600 \mu \mathrm{m}, 2 a=144 \mu \mathrm{m}, L=500 \mu \mathrm{m}$, and wavelength $\lambda_{o}$ $=980 \mathrm{~nm}$. First, the results in Fig. 5(a) employ the nominal 
grating distance $l=3904 \mu \mathrm{m}$ inside Eq. (17) instead of the effective $\hat{l}$ and $\bar{l}$. A maximum overlap strength $V_{12} \simeq 1 / 2$ (half the mode power is emitted in each sidelobe) occurs for $n_{o}=12$ and waist size $w_{o} \simeq 60 \mu \mathrm{m}$. The coupling strength involving the nearby transverse modes $n_{o} \pm 1$ or $n_{o} \pm 2$ is reduced by a factor of 2.5-3 because the off-set between the reflected sidelobe center and the cavity center is comparable to the mode waist $w_{o}$. Next, the results in Fig. 5(b) employ the correct effective values: an effective $\hat{l}=3904 \mu \mathrm{m}$ corresponds to the same resonant $n_{o}=12$ mode for an actual grating distance $l=3291 \mu \mathrm{m}$. The corresponding diffraction length $\bar{l}$ varies as a function of $w_{o}$ and $\theta_{n}$. The maximum overlap strength $V_{12}$ is reduced to 0.35 due to the distributed interaction (i.e., additional diffraction) inside the grating. Still, the coupling strength for the nearby transverse modes is reduced by a factor of 2 relative to the $n_{o}$ mode, hence, the array geometry still leads to transverse mode selection.

\section{COUPLED MODE GAIN}

The goal now is to demonstrate that modes coupled via sidelobe reflection by the grating have much higher growth rate than free running modes in isolated cavities. First, we will demonstrate the case for two coupled cavities and then for an arbitrary size array.

\section{A. Two coupled cavities}

For operation near threshold current $I \geq I_{\text {th }}$, or during the initial low power, exponential growth stage, radiative depletion in the carrier density equation can be neglected compared to nonradiative recombination. One may then assume a uniform, near threshold carrier density $N_{j} \simeq N_{\text {th }}$ and a uniform cavity gain $g_{j}=g$. The matrix equation for the growth rate Eq. (12) becomes

$$
\begin{aligned}
\frac{\partial}{\partial t}\left(\begin{array}{l}
\mathcal{E}_{1} \\
\mathcal{E}_{2}
\end{array}\right) & =\gamma\left(\begin{array}{l}
\mathcal{E}_{1} \\
\mathcal{E}_{2}
\end{array}\right) \\
& =\left[\begin{array}{cc}
v_{g}\left(g_{1} \Upsilon_{11}-\mu_{1} M_{11}\right) & v_{g} \nu V_{12} \\
v_{g} \nu V_{12}^{*} & v_{g}\left(g_{2} \Upsilon_{22}-\mu_{2} M_{22}\right)
\end{array}\right],
\end{aligned}
$$

where we have allowed slightly different cavity parameters $g_{1,2}, \mu_{1,2}$ to reflect manufacturing tolerances between cavities. Defining $G_{1}=\left(g_{1} \Upsilon_{11}-\mu_{1} M_{11}\right), G_{2}=\left(g_{2} \Upsilon_{22}-\mu_{2} M_{22}\right)$ the growth constant $\Gamma=\gamma / v_{g}$ for the coupled cavity modes is given by the roots of the determinant

$$
\begin{aligned}
\left|\begin{array}{cc}
G_{1}-\Gamma & \nu V_{12} \\
\nu V_{12}^{*} & G_{2}-\Gamma
\end{array}\right|= & 0 \Rightarrow \Gamma_{ \pm}=\frac{G_{1}+G_{2}}{2} \\
& \pm \frac{\sqrt{\left(G_{1}-G_{2}\right)^{2}+4 \nu^{2}\left|V_{12}\right|^{2}}}{2}
\end{aligned}
$$

while the cavity amplitude ratio $\left(\mathcal{E}_{2} / \mathcal{E}_{1}\right)_{ \pm}=\left(G_{1}-\Gamma_{ \pm}\right) / \nu V_{12}$. For strongly coupled modes $\left|V_{12}\right|>\left|G_{1}-G_{2}\right|$ we have $\Gamma_{ \pm}$ $\simeq\left[\left(G_{1}+G_{2}\right) / 2\right] \pm\left|\nu V_{12}\right|$ and the highest growth rate $\Gamma_{+}$occurs for the mode with $\mathcal{E}_{2} / \mathcal{E}_{1} \simeq-V_{12} /\left|V_{12}\right|=-e^{i \Psi_{12}}$ with $\Psi_{12}$ being the phase advance along the feedback optical path. When $\nu\left|V_{12}\right|>\left|G_{1}-G_{2}\right|$ the coupled growth rate $\Gamma_{+}$exceeds the growth rate of either free running mode $G_{1,2}$; in fact, the exact eigenvalue $\Gamma_{+}$always exceeds the highest of the free running $\max \left(G_{1}, G_{2}\right)$. Now, reviving the implied modal dependence of the coupling coefficient, the coupling is selective and applies only to free running modes of given $\left(k_{x}, k_{z}\right)=(n \pi / a, p \pi / L), \quad$ i.e., $\quad V_{12}=V_{12}\left(n^{\prime}, p^{\prime}\right) \delta\left(n^{\prime}-n\right) \delta\left(p^{\prime}\right.$ $-p)$. Since the coupled cavity modes have higher growth rates than the corresponding free running modes, and since only one coupled mode exists with proper selection of PTR reflectivity and cavity geometry, this implies simultaneous phase locking and mode selection. The effect is made stronger by the low reflectivity $(5 \%-10 \%)$ of the AR coating, causing high cavity losses that exceed material gain $\mu_{1,2}$ $\gg g_{1,2}$ under usual current biases. As a result, free running modes remain below threshold $G_{1,2}<0$ and only the coupled mode, experiencing the high reflectivity of the grating $\nu\left|V_{12}\right|>-\left(\mu_{1}+\mu_{2}\right) / 2$, exceeds lasing threshold.

Mode locking of two multimode emitting free-running lasers into a single mode operation has been demonstrated in Ref. 6 via the use of external grating. The phase locking into single mode occurs for grating reflection bandwidth of 40 $\mathrm{nm}$, smaller than the longitudinal mode separation of $70 \mathrm{pm}$ (the transverse mode separation is about $150 \mathrm{pm}$ ). Single mode coupling among three laser diodes has also been achieved.

\section{B. Periodic array}

The discussion is extended to an arbitrary size periodic array of identical cavity parameters. Taking advantage of the symmetric tridiagonal form of Eq. (12) we introduce the transformation $\mathcal{E}_{j}(t)=e^{i(j \Phi)} \hat{\mathcal{E}}_{o}(t)$ and search for eigenvalues $\left(1 / v_{g}\right)(\partial / \partial t) \hat{\mathcal{E}}_{o}=\Gamma \hat{\mathcal{E}}_{o}$ casting Eq. (12) in the form

$$
\left(\begin{array}{ccccc}
G-\Gamma & \nu\left|V_{12}\right| e^{i\left(\Psi_{12}+\Phi\right)} & 0 & \ldots & \ldots \\
\nu\left|V_{12}\right| e^{i\left(\Psi_{12}-\Phi\right)} & G-\Gamma & \nu\left|V_{12}\right| e^{i\left(\Psi_{12}+\Phi\right)} & 0 & \ldots \\
\ldots & \ldots & \ldots & \ldots & \ldots \\
0 & \nu\left|V_{12}\right| e^{i\left(\Psi_{12}-\Phi\right)} & G-\Gamma & \nu\left|V_{12}\right| e^{i\left(\Psi_{12}+\Phi\right)} & 0 \\
\ldots & \ldots & \ldots & \ldots & \ldots \\
\ldots & 0 & 0 & \nu\left|V_{12}\right| e^{i\left(\Psi_{12}-\Phi\right)} & G-\Gamma
\end{array}\right)\left(\begin{array}{c}
\hat{\mathcal{E}}_{o} \\
\hat{\mathcal{E}}_{o} \\
\ldots \\
\hat{\mathcal{E}}_{o} \\
\ldots \\
\hat{\mathcal{E}}_{o}
\end{array}\right)=0
$$


where $\Gamma=\gamma / v_{g}$ and, due to identical parameters, $G=g_{o} \Upsilon_{11}$ $-\mu_{o} M_{11}$ and $V_{12}=\left|V_{12}\right| e^{i \Psi_{12}}$. Defining $\Theta=\Phi+\Psi_{12}$ and $Z$ $=(G-\Gamma) /\left(\nu\left|V_{12}\right| e^{i \Psi_{12}}\right)$ recasts the left-hand matrix as $\nu\left|V_{12}\right| \mathcal{I} \mathcal{Z}$, where

$$
\mathcal{Z}=\left(\begin{array}{ccccc}
Z & e^{i \Phi} & 0 & \cdots & \cdots \\
e^{-i \Phi} & Z & e^{i \Phi} & 0 & \cdots \\
\cdots & e^{-i \Phi} & Z & e^{i \Phi} & \cdots \\
\cdots & \cdots & \cdots & \cdots & \cdots \\
\cdots & 0 & 0 & e^{-i \Phi} & Z
\end{array}\right)
$$

The eigenvalue equation is given by $\mathcal{D} \equiv \operatorname{det} \mathcal{Z}=0$. The determinant satisfies the recursion relation $\mathcal{D}_{N+1}=Z \mathcal{D}_{N}-\mathcal{D}_{N-1}$ relative to the array size $N$. Since any equation line yields the relation $Z=2 \cos \Phi$, the determinant recurrence relation is satisfied by by $\mathcal{D}_{N}=A \sin [(N+1) \Phi]=\sin [(N+1) \Phi] / \sin \Phi(A$ selected so as to satisfy $\left.\mathcal{D}_{1}=A \sin 2 \Phi=2 \cos \Phi\right)$. The dispersion $\mathcal{D}_{N}=0$ yields $\Phi_{q}=q \pi /(N+1)$ with $q=1,2, \ldots, N$ (but $q \neq 0, N+1)$. From the definitions of $Z \equiv(G-\Gamma) /|V|=$ $-2 \cos \Phi$ follows the gain eigenvalues:

$$
\Gamma_{q}=G+2 \nu\left|V_{12}\right| \cos \Psi_{12} \cos \Phi_{q} .
$$

For in-phase optical path $\Psi_{12}=2 l \pi$ with $\cos \Psi_{12}>0$ the collective coupled mode gain exceeds the free running gain $G$ for $0<\Phi_{q}<\pi / 2$; the highest growth occurs for the "inphase" mode $\Phi=\pi /(N+1)$. For out-of-phase optical path $\Psi_{12}=(2 l+1) \pi$ with $\cos \Psi_{12}<0$ the highest growth corresponds to "out-of-phase" cavity phasing $\Phi=N \pi /(N+1)=\pi$ $-\pi /(N+1)$.

According to Eqs. (21) and (22), the fastest growing mode $\Gamma_{+}$for two identical $G_{1}=G_{2}$ in-phase $\mathcal{E}_{1} / \mathcal{E}_{2}=1$ coupled cavities corresponds to a feedback phase-advance $\Psi=\pi$ and is given by

$$
\Gamma=g_{1} \Upsilon_{11}-\mu_{1} M_{11}+\nu\left|V_{12}\right| \text {. }
$$

A similar maximum growth formula is obtained for an inphase $\Phi=0$ array Eq. (25) for $\Psi_{12} \simeq \pi$, implying again a feedback phase $\Psi_{12} \simeq \pi$. Here each cavity receives feedback from two neighbors so $\nu\left|V_{12}\right| \rightarrow 2 \nu\left|V_{12}\right|$ applies in Eq. (26). One can then determine the threshold gain, from $\Gamma=0$, as

$$
\hat{g}_{1}=\mu_{1} \frac{M_{11}}{\Upsilon_{11}}-2 \nu \frac{\left|V_{12}\right|}{\Upsilon_{11}} .
$$

The coupling reduces the gain threshold as radiation feedback from the grating reflection makes up for edge cavitymirror losses. Since the feedback strength depends on the sidelobe angle $\theta_{n}$, coupling diminishes and threshold increases as one moves away from the optimum coupling angle $\tan \theta_{n} \equiv(n \pi / a) / k_{o}=d / 2 l$, corresponding to transverse wavenumber $n_{o}=\left[k_{o} a d / 2 \pi l\right]([\cdots]$ stands for integer part). Figure 4 shows the collective mode threshold versus transverse mode wavenumber for typical parameters $R_{c}=0.10, \Upsilon_{11}$ $=0.10, M_{11}=0.90$, and $R_{G}=0.99$, with the same array geometry as in Fig. 3, corresponding to $n_{o}=12$. For the array eigenmodes involving coupled $n_{o}+1$, or coupled $n_{o}-1$, transverse modes the threshold gain increases by a factor of 3 ; for $n$ far from $n_{o}$ the coupling strength is practically zero and one recovers the threshold of the "free running" cavity modes; this threshold is higher by a factor of 5 from the $n_{o}$
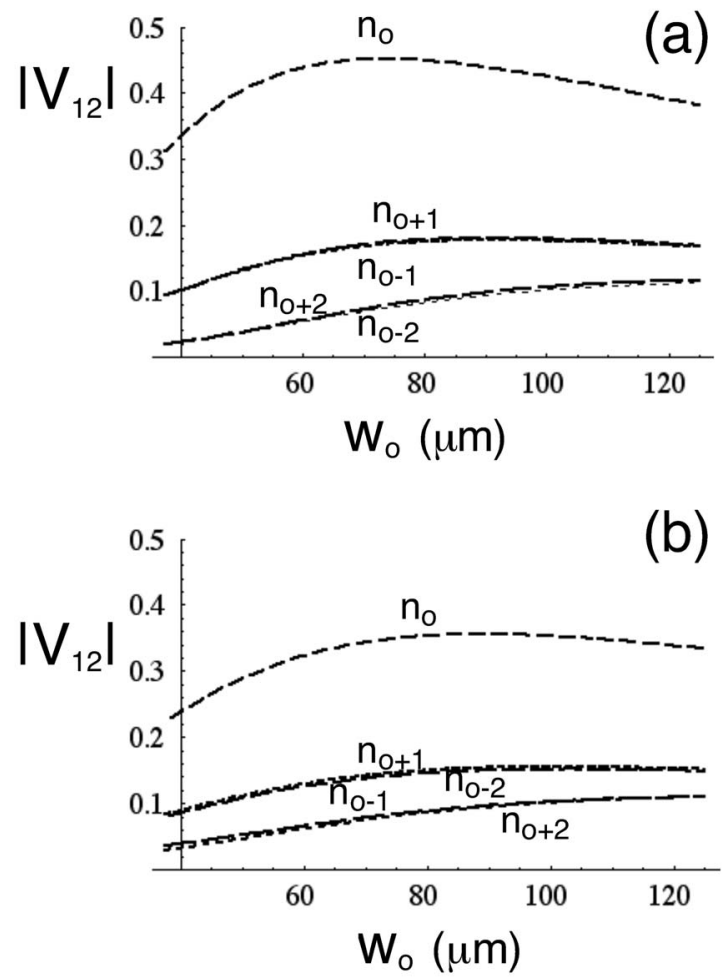

FIG. 6. (a) Coupling (overlap) strength vs cavity mode waist, for various transverse wavenumbers (a) Using the actual grating distance $l=3904 \mu \mathrm{m}$ in the coupling formula (b) using an effective distance $\hat{l}=3904 \mu \mathrm{m}$ (corresponding to an actual $l=3291 \mu \mathrm{m}$ ) and the related diffraction distance $\bar{l}$, to account for the distributed grating interaction. The other array parameters used are, $d=600 \mu \mathrm{m}, 2 a=144 \mu \mathrm{m}, L=500 \mu \mathrm{m}$, and wavelength $\lambda_{o}$ $=980 \mathrm{~nm}$. In both cases maximum coupling occurs for transverse mode number $n_{o}=12$ corresponding to $\theta_{n}=4.497^{\circ}$.

coupled-cavity mode because the AR reflectivity is much lower than the grating.

\section{VERTICAL MODE SUPPRESSION}

Due to the finite frequency width $D \omega$ of the material gain $g(\omega)>0$ many modes of the same longitudinal $k_{z}=k_{p}$ but different transverse $k_{n}$ wavenumber experience positive material gain, Fig. 6(a). The vertical free running mode $n$ $=0$ with $k_{x}=k_{0}=\theta_{0}=0$ stands out because it experiences direct reflection from the grating and thus high per-pass amplification, competing with coupled modes. One approach for removing the vertical mode is to tailor the center wavenumber $k_{z}$ reflected from the grating so that the frequency $\omega\left(k_{z}, k_{n=0}\right)$ lies just below the positive material gain, Fig. 6(b). Then only oblique modes with $k_{x}=k_{n \neq 0}$ fall in the positive material gain region, $g\left[\omega\left(k_{z}, k_{n=0}\right)\right]<0<g\left[\omega\left(k_{z}, k_{n \neq 0}\right)\right]$. The oblique free running modes are subthreshold and only the coupled mode is excited. Another method would be to spatially erase (actually detune) the grating directly above the cavities, Fig. 6(b), so that negligible reflection occurs for vertical incidence within the positive gain region.

Perhaps the simplest approach is based on the observation that the vertical mode experiences multiple reflections between the grating and the AR coating; in effect the AR and the grating form an external resonator for the vertical mode. 

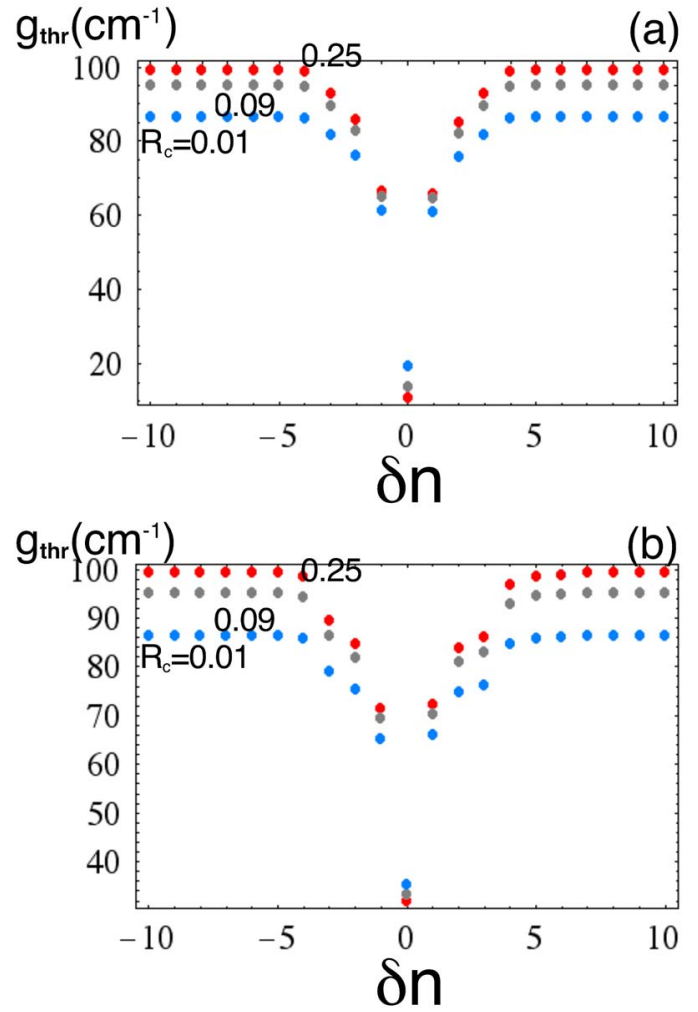

FIG. 7. (Color online) Collective array mode gain threshold vs transverse mode number shift from the optimum, $\delta n=n-n_{o}$, for typical grating reflectivity $R_{G}=0.99$, gain confinement factor $\Upsilon_{11}=0.10$, and edge mirror confinement factor $M_{11}=0.90$. Same array geometry as in Fig. 3, corresponding to $n_{o}=12$. (a) Using actual grating separation and neglecting the distributed interaction effects inside the grating (b) including the internal grating diffraction via the use of effective distances. Various colors correspond to different $\mathrm{AR}$ coating reflectivities $R_{c}$ as marked.

Etalon theory shows that the combined reflection coefficient is determined by interference effects and given by

$$
\begin{aligned}
\left|R_{G C}\right|^{2} & =\left|\frac{R_{c}+V_{11}\left|R_{G}\right| e^{i \Psi_{o}}}{1-V_{11}\left|R_{G}\right| R_{c} e^{i \Psi_{o}}}\right|^{2} \\
& =\frac{\left(R_{c}+V_{11}\left|R_{G}\right|\right)^{2}-4 V_{11}\left|R_{G}\right| R_{c} \sin ^{2} \frac{\Psi_{o}}{2}}{\left(1+V_{11}\left|R_{G}\right| R_{c}\right)^{2}+4 V_{11}\left|R_{G}\right| R_{c} \sin ^{2} \frac{\Psi_{o}}{2}},
\end{aligned}
$$

where the round trip phase, given by

$$
\Psi_{o}=2 k_{o} \hat{l}+\chi_{o}-\varphi(2 \bar{l})
$$

contains the phase corrections from the grating reflection $\chi_{o}$ and the paraxial diffraction $\varphi$ relative to plane wave optical path $2 k_{o} \hat{l}$. The factor $V_{11}$ is the "self-interference" factor expressing the overlap of the reflected, expended wavefront with the original Gaussian; $V_{11} \rightarrow 1$ for plane infinite wave (i.e., zero diffraction) in the usual etalon approximation. The vertical reflectance $\left|R_{V}\right|^{2}$ plotted in Fig. 7 is much smaller than either the AR coating $\left|R_{c}\right|^{2}$ or the grating reflectance $\left|R_{G}\right|^{2}$ if the round trip path equals odd number of halfwavelengths $\Psi_{o}=(2 q+1) \pi$, for destructive interference. Hence, the combined reflectivity for the vertical mode in this case is reduced to the level of the AR coating reflectivity. No multiple reflections occur for the oblique sidelobe modes

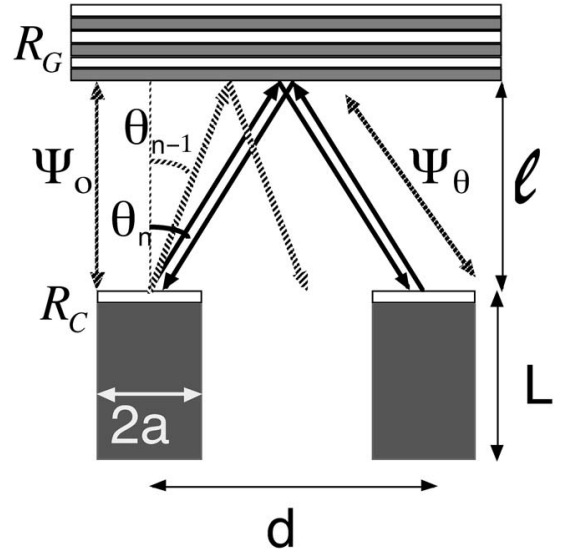

FIG. 8. Geometric illustration of the phase advances for the vertical mode, and for the oblique mode coupling

where the intercavity reflection coupling is still given by the single-pass $\left|R_{12}\right|=\left|V_{12}\right| R_{G}\left(1-R_{c}^{2}\right) \simeq\left|V_{12}\right| R_{G}$.

The effective grating separation $\hat{l}$ can now be chosen to simultaneously satisfy the destructive interference condition for the vertical mode $\Psi_{o}=(2 q+1) \pi$ and the in-phase locking condition for the coupled array mode $\Psi_{\theta}=\left(2 q^{\prime}+1\right) \pi$, respectively,

$$
\begin{aligned}
& 2 k_{o} \hat{l}+\chi_{o}-\frac{1}{2} \varphi(2 \bar{l})=(2 q+1) \pi, \\
& 2 k_{o}^{\prime} \hat{l}+\chi_{\theta}-\left[\varphi(2 \bar{l})-\frac{1}{2} \varphi(\bar{l})\right]+\frac{k_{o}^{\prime} X_{\theta}\left(X_{\theta}+2 \delta\right)}{4 \hat{l}} \\
& =\left(2 q^{\prime}+1\right) \pi,
\end{aligned}
$$

where $k_{o}=\omega / c, X_{\theta} \equiv 2 \hat{l} \tan \theta$, and $\delta=d-2 \hat{l} \tan \theta$ is the offset between the reflected wavefront and the cavity center. According to the previous section different effective lengths apply for the effective reflection length (axial path) $\hat{l}$ and the diffraction length $z=2 \bar{l}$ carried inside $\varphi(z)=\tan ^{-1}(z / b)$ $=\tan ^{-1}\left[2 z /\left(k_{o} w_{o}\right)^{2}\right]$. The paraxial limit $\sin \theta \simeq \tan \theta$ applies. For a given $k_{z}^{o}$, fixed by the grating period we have $k_{o}=k_{z}^{o}$ for the vertical mode and $k_{o}^{\prime}=k_{o} \sqrt{1+\tan ^{2} \theta}$ for sidelobe modes. Subtracting Eq. (31) from Eq. (30) yields

$$
\begin{aligned}
2\left(k_{o}-k_{o}^{\prime}\right) \hat{l}_{q}+\chi_{o}-\chi_{\theta}-\frac{1}{2} \tan ^{-1}\left[\frac{2 k_{o}^{\prime} \hat{l}_{q}}{\left(k_{o}^{\prime} w_{o}\right)^{2}}\right] \\
-k_{o}^{\prime} \hat{l}_{q}\left(\tan ^{2} \theta+\frac{k_{o}^{\prime} \delta}{k_{o}^{\prime} l_{q}} \tan \theta\right)=2\left(q-q^{\prime}\right) \pi .
\end{aligned}
$$

Calling the left-hand side $\Psi_{q}$, Eq. (32) is equivalent to

$$
\bmod \left(\Psi_{q}, 2 \pi\right)=0,
$$

where $k_{o} \hat{l}_{q}$ is given by Eq. (30). Equations (30), (32), and (33) admit integer solutions $q$ only for specific values of $\theta_{q}$. The corresponding effective grating distance $\hat{l}_{q}=d / 2 \sin \theta_{q}$ follows directly from the zero off-set $\delta=0$ requirement, between the reflected wavefront center and the next cavity center; this relation is included upfront inside Eq. (32). Graphic solutions are shown in Figs. 8(a) and 8(b), plotting, respec- 

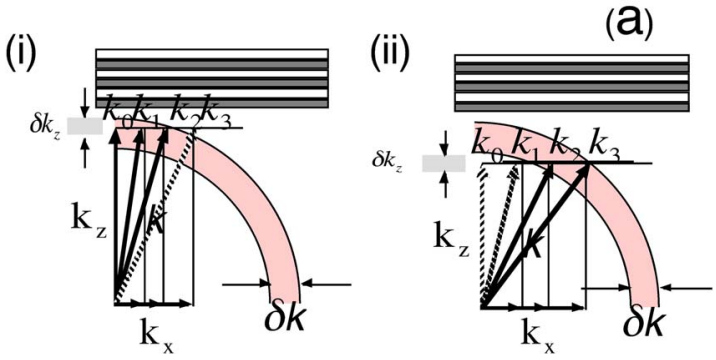

(b)

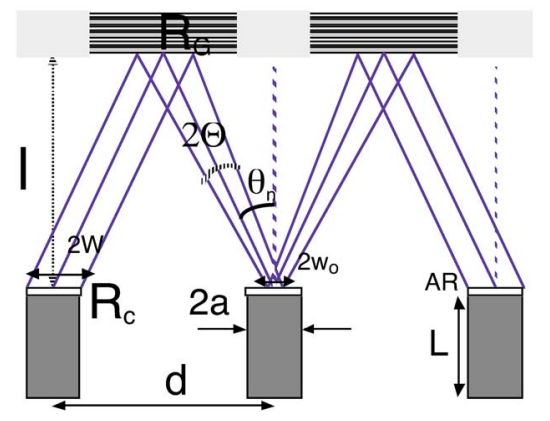

FIG. 9. (Color online) (a) Wavenumber control by the grating and by the material gain. Circular ring denotes the frequency range with positive material gain: (left) positive gain modes of same $k_{z}$ include the vertical mode $k=k_{z}, k_{x}=0$ (right) Vertical mode not in the positive gain region. (b) Use of a spatially nonuniform DBR grating with erased or detuned regions above the cavities, that shift the reflection $k_{z}$ window for vertical incidence.

tively, $\operatorname{Mod}\left(\Psi_{q}\right)_{2 \pi}$ versus $\hat{l}_{q}$ and $\operatorname{Mod}\left(\Psi_{q}\right)_{2 \pi}$ versus $\theta_{q}$; the sought-after values $\hat{l}_{q}$ and $\theta_{q}$ are given by the zero intercepts $\operatorname{Mod}\left(\Psi_{q}\right)_{2 \pi} \simeq 0$ (Figs. 9-11). Thus satisfying both destructive vertical interference and in-phase oblique cavity coupling yields a set of discrete values $l_{q}$ and $\theta_{q}$ for the grating distance, and the related sidelobe angle, under given cavity separation $d$. One such example is $q=7075, \hat{l}_{q}=3533 \mu \mathrm{m}$, and $\theta_{q}=4.78^{\circ}(q+1 / 2$ being the approximate value of the grating separation in half-wavelengths.) Since $\theta_{q}$ must also coincide with the sidelobe angle for some transverse cavity mode, $\tan \theta_{q}=\tan \theta_{n} \equiv k_{n} / k_{o}=(n \pi / a) / k_{o}$, it follows that $n(q)=\tan \theta_{q} k_{o} a / n \pi$. The cavity width $2 a$ can now be selected so that the $n(q)$ values are integer numbers. For $\theta_{q}$ $=4.78^{\circ}$ we get $n=13$ by letting $2 a=144 \mu \mathrm{m}$ [for the same given $a$, there are other $\theta_{q}$ values in Fig. 8(b) yielding integer $n$ in Fig. 8(c)].

In conclusion the analysis shows that transverse mode selection and phase locking is possible in edge emitting arrays via sidelobe reflection coupling off a narrow bandwidth grating, with concurrent suppression of vertical mode lasing. The analysis assumed "ideal" arrays of identical cavity parameters. In practice, unavoidable manufacturing variations and thermal gradients cause variations from the nominal lasing frequencies. Phase locking is then possible only when the nonlinear frequency pulling caused by cavity coupling can overcome the original variations in the cold-cavity frequencies. A relation exists ${ }^{9}$ between the rms cavity-to-cavity variations, the maximum array size $N$ and the coupling strength $V_{12}$, limiting scalability of the array size. This issue will be addressed further in future work.
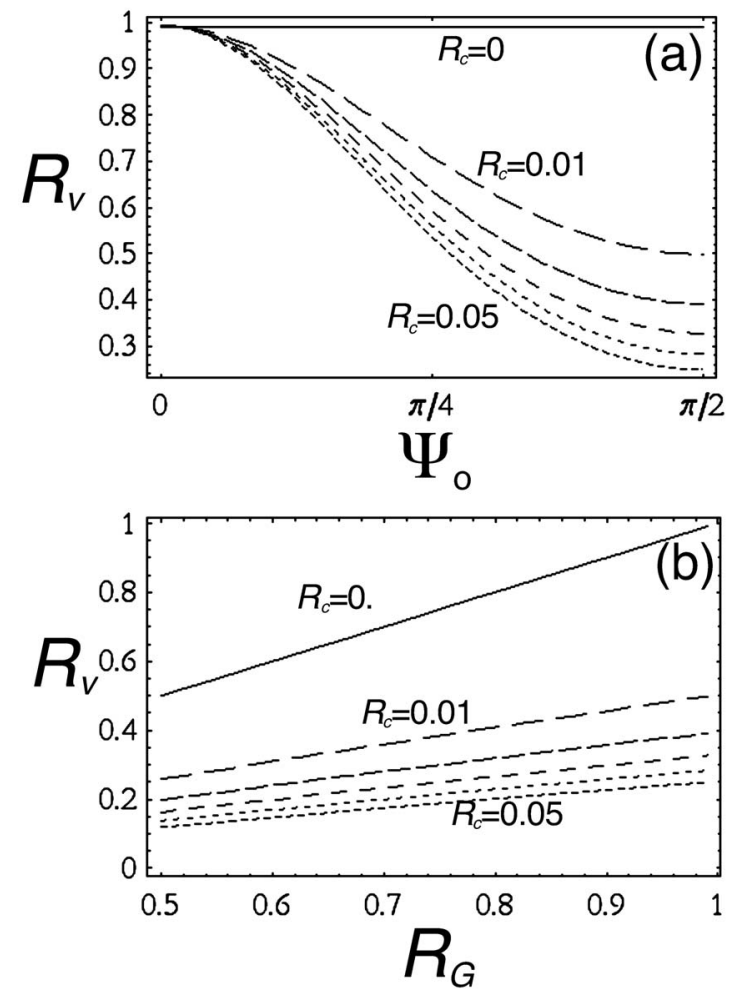

FIG. 10. (a) The vertical mode reflectance $\mathcal{R}_{V}=\left|R_{V}\right|^{2}$ vs optical path phase, involving interference between the grating and the coating reflections (etalon effect). The grating reflectance is $\mathcal{R}_{G}=\left|R_{G}\right|^{2}=0.99$ and various curves represent different AR coating reflectance $\mathcal{R}_{C}=\left|R_{C}\right|^{2}$ as marked. (b) For destructive interference $\Psi_{o}=\pi / 2$, the combined vertical reflectance is much lower than the grating reflectance. In both (a) and (b) $\mathcal{R}_{V}$ decreases with increasing coating reflectance.

\section{APPENDIX A: DERIVATION OF COUPLED-CAVITY EQUATIONS}

In Fig. 1 the active cavity regions are located between $-L<z<0$, where $R_{o}$ is the output mirror reflectivity at $z=0$, $R_{-L}$ is the backmirror reflectivity at $z=-L$, and $R_{G}$ is the grating mirror reflectivity placed at $z=l$. We now separate the circulating cavity radiation in forward and backward waves $\propto e^{ \pm k_{z} z}$ with slowly varying envelopes $\mathcal{E}^{ \pm}(z, t) \mathcal{U}(\mathbf{r})$. From now on the time dependence is implied inside $\mathcal{E}$. Integrating over the array volume, and since for guided mode profiles $z, \mathbf{r}$ are separable variables

$$
\begin{gathered}
\int_{-L}^{0} d z\left[\frac{\partial}{\partial t} \mathcal{E}^{ \pm}(z)+v_{g}^{ \pm} \frac{\partial}{\partial z} \mathcal{E}^{ \pm}(z)\right] \int d \mathbf{r}^{2} \mathcal{U}(\mathbf{r}) \\
=g \int_{-L}^{0} d z^{ \pm}(z) \int d \mathbf{r}^{2} \xi^{g}(\mathbf{r}) \mathcal{U}(\mathbf{r}) .
\end{gathered}
$$

Letting $v_{g}^{ \pm}(\partial / \partial z) \mathcal{E}^{ \pm}=(\partial / \partial z)\left(v_{g}^{ \pm} \mathcal{E}^{ \pm}\right)$for constant group velocity inside the cavity and applying Stokes identity converts the $\partial / \partial z$ integrant into a surface integral over the array edge areas at $z=-L$ and $z=0$, 

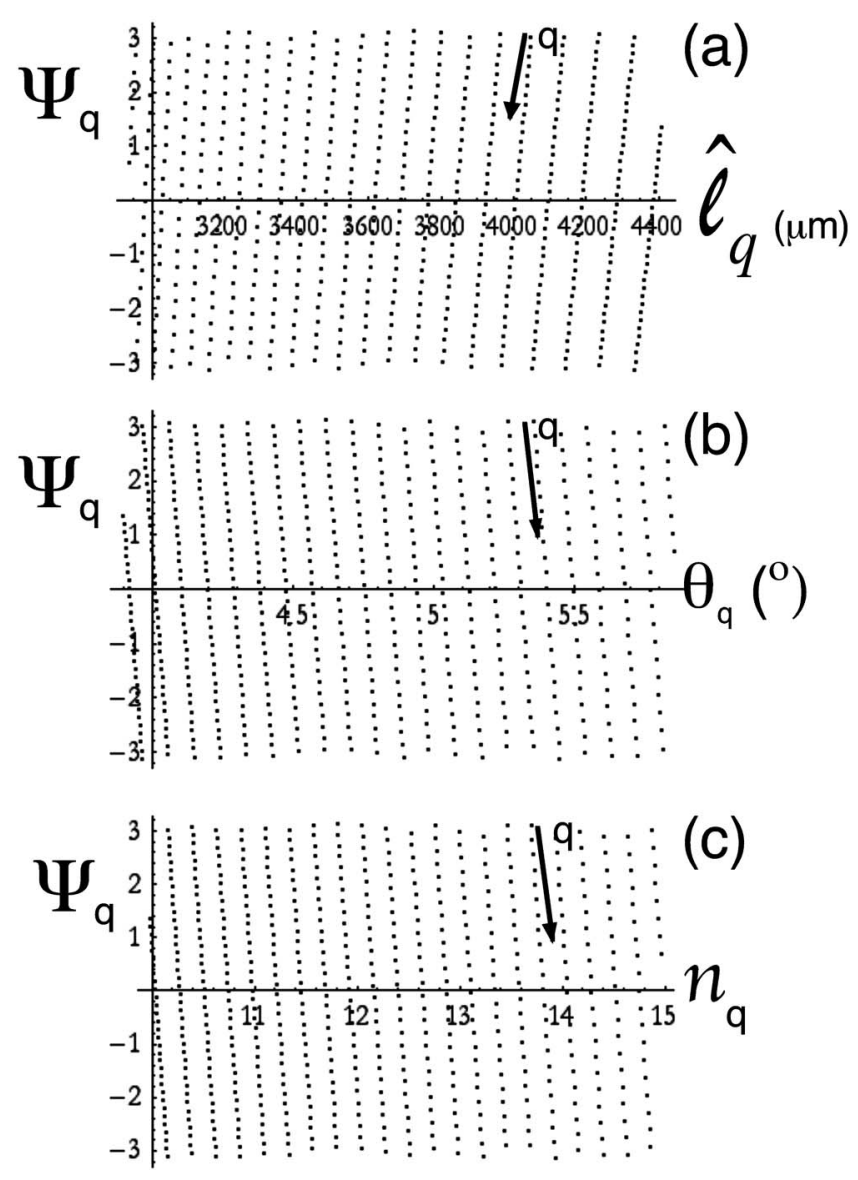

FIG. 11. Geometrical design parameters for simultaneous vertical mode suppression and in-phase oblique cavity phase locking. The corresponding effective grating distances $\hat{l}_{q}$ in (a), and the sidelobe angles $\theta_{q}$ in (b) are given by the horizontal axis intersections $\operatorname{Mod}\left(\Psi_{q}\right)_{2 \pi}=0$. Some of these intersections also yield integer transverse mode numbers $n(q)$ in (c). The other array parameters are $d=600 \mu \mathrm{m}, 2 a=144 \mu \mathrm{m}, w_{o}=75 \mu \mathrm{m}, L$ $=500 \mu \mathrm{m}$, and wavelength $\lambda_{o}=980 \mathrm{~nm}$. Each dot shows a $q$ value differing by unity, and arrows point the increasing direction, from 6000 to 9000 .

$$
\begin{aligned}
& \int_{-L}^{0} d z \frac{\partial}{\partial t} \mathcal{E}^{ \pm}(z) \int d \mathbf{r}^{2} \mathcal{U}(\mathbf{r}) \pm \int_{S} d \mathbf{r}^{2} v_{g}^{ \pm} \mathcal{E}^{ \pm}(0) \mathcal{U}(\mathbf{r}) \\
& \mp \int_{S} d \mathbf{r}^{2} v_{g}^{ \pm} \mathcal{E}^{ \pm}(-L) \mathcal{U}(\mathbf{r}) \\
& =g \int_{-L}^{0} d z \mathcal{E}^{ \pm}(z) \int d \mathbf{r}^{2} \chi(\mathbf{r}) \mathcal{U}(\mathbf{r})
\end{aligned}
$$

The surface terms give the radiation flux escaping the cavities in the outward direction at $z=0$ and $z=-L$, respectively, $v_{g}^{ \pm} \hat{\mathbf{z}}= \pm v_{g}$ and $v_{g}^{ \pm}(-\hat{\mathbf{z}})=\mp v_{g}$. For the right going wave the surface terms yield the output cavity losses

$$
\int_{S} d \mathbf{r}^{2} v_{g} T_{o} \mathcal{E}^{+}(0) \mathcal{U}(\mathbf{r})
$$

For the left going wave the surface terms yield the backmirror losses, and the feedback influx at $z=0$ from the external cavity grating reflection

$$
\int_{S} d \mathbf{r}^{2} v_{g} T_{b} \mathcal{E}^{-}(-L) \mathcal{U}(\mathbf{r})-\int_{S} d \mathbf{r}^{2} v_{g} R_{F} \mathcal{P} \mathcal{E}^{+}(0) \mathcal{U}(\mathbf{r} \pm \mathbf{d})
$$

where $R_{F}=T_{o} R_{G} T_{o}$ and $\mathcal{P}_{z=2 l} \mathcal{E}^{+}(0) \mathcal{U}(\mathbf{r} \pm \mathbf{d})$ produces the diffracted radiation wavefronts emanating from neighbor cavities, $\mathcal{P}_{z=2 l}$ being the paraxial propagator over twice the grating distance $2 l$. Assuming bidirectional gain $\mathcal{E}^{+}(z)$ $=\mathcal{E}^{+}(-L) e^{\kappa(z+L)}$ and $\mathcal{E}^{-}(z)=\mathcal{E}^{-}(0) e^{-\kappa z}$ the integral along the cavity length yields

$$
\begin{aligned}
& \mathcal{O} \int_{-L}^{0} d z \mathcal{E}^{+}(z)=\mathcal{O} L \mathcal{E}^{+}(-L) \frac{e^{\kappa L}-1}{\kappa L}=\mathcal{O} L \mathcal{E}^{+}(0) \frac{1-e^{-\kappa L}}{\kappa L}, \\
& \mathcal{O} \int_{-L}^{0} d z \mathcal{E}^{-}(z)=\mathcal{O} L \mathcal{E}^{-}(0) \frac{e^{\kappa L}-1}{\kappa L}=\mathcal{O} L \mathcal{E}^{-}(-L) \frac{1-e^{-\kappa L}}{\kappa L},
\end{aligned}
$$

where $\mathcal{O}$ is any scalar [i.e., $\chi(\mathbf{r})]$ or operator (i.e., $\partial / \partial t$ ) that does not depend on $z$. The effective cavity length is defined by $2 \hat{L} \mathcal{E}^{+}(0) \equiv \int_{-L}^{0} d z \mathcal{E}^{+}(z)+\int_{-L}^{0} d z \mathcal{E}^{-}(z)$ yielding, together with the boundary condition $\mathcal{E}^{+}(-L)=R_{-L} \mathcal{E}^{-}(-L)=\mathcal{E}^{+}(0) e^{-\kappa L}$,

$$
\hat{L}=L \frac{1-e^{-\kappa L}}{\kappa L} \frac{1+R_{-L}}{2 R_{-L}} .
$$

The effective $\hat{L}$ accounts for the amplitude variation along the cavity; $\hat{L} \rightarrow L$ for small gain $\kappa L \ll 1$ and $R_{-L} \simeq 1$. Summing up the volume integrals for both right- and left-handed waves, which amounts to a volume integral over one round trip, and inserting expressions (A2), (A3), and (A7) yields

$$
\begin{aligned}
\frac{\partial}{\partial t} \mathcal{E}^{+}(0) \int d \mathbf{r}^{2} \mathcal{U}(\mathbf{r})= & g \mathcal{E}^{+}(0) \int d \mathbf{r}^{2} \mathcal{U}(\mathbf{r}) \zeta^{g}(\mathbf{r}) \\
& -\frac{T_{o}}{2 \hat{L}} \mathcal{E}^{+}(0) \int d \mathbf{r}^{2} \mathcal{U}(\mathbf{r}) \zeta^{m}(\mathbf{r}) \\
& -\frac{T_{-L}}{2 \hat{L}} \mathcal{E}^{-}(-L) \int d \mathbf{r}^{2} \mathcal{U}(\mathbf{r}) \zeta^{m}(\mathbf{r}) \\
& +\frac{R_{F}}{2 \hat{L}} \mathcal{E}^{+}(0) \int d \mathbf{r}^{2} \mathcal{U}(\mathbf{r}) \mathcal{P}_{z=2 l} \mathcal{U}(\mathbf{r} \pm \mathbf{d})
\end{aligned}
$$

One may substitute $\mathcal{E}^{-}(-L)=\mathcal{E}^{+}(0) e^{-\kappa L} / R_{-L}$, whereby Eq. (A8) expresses the time evolution of the right-propagating wave amplitude at the output $\mathcal{E}^{+}(0)$. We now unfold the implicit periodic superposition for the array mode profile, gain and reflectivity inside Eq. (A8), according to $\mathcal{E}(z) \mathcal{U}(\mathbf{r})$ $=\Sigma_{j} \mathcal{E}_{j}(z) U_{j}(\mathbf{r})=\Sigma_{j} \mathcal{E}_{j}(z) U(\mathbf{r}-j d), \quad \zeta^{m, g}(\mathbf{r})=\Sigma_{j} \zeta_{j}^{m, g}(\mathbf{r})$ $=\sum_{j} \zeta^{m, g}(\mathbf{r}-j d)$. Taking the projection of Eq. (A8) with the $i$ th cavity mode profile, and using the coupling coefficient definitions Eqs. (6)-(8) yields the rate Eq. (5) for the slow time evolution of the $i$ th cavity complex envelope at the output $\mathcal{E}_{i}(t) \equiv \mathcal{E}_{i}^{+}(z=0, t)$. 
The cavity loss coefficients at the cavity edges must be computed from the definitions of power reflectance $R$ and transmittance $T$, where $P_{r}=R P_{o}$ and $P_{t}=T P_{o}$ are, respectively, the reflected and transmitted power at a given interface. The reflected and transmitted amplitudes for an incoming monochromatic wave of amplitude $E_{o}$ incident at an angle $\theta$ at the interface between dielectric constants $\epsilon, \epsilon^{\prime}$ are given by $E_{o}^{\prime}=r E_{o}$ and $E_{o}^{\prime \prime}=t E_{o}$, respectively. The amplitude reflectivity and transmissivity, respectively, $r, t$, follow from boundary condition matching. Substituting these values in the general expression for the power flux $\left(v_{g} / 16 \pi\right)\left(\epsilon^{2} E_{o}^{2}\right.$ $\left.+B_{o}^{2}\right)=v_{g} / 16 \pi\left(\epsilon E_{o}^{2}+(c k / \omega)^{2} E_{o}^{2}\right)=\left(v_{g} \varepsilon / 8 \pi\right) E_{o}^{2}$, the power balance $P_{o}=P_{R}+P_{T}$ yields

$$
\cos \theta \frac{v_{g} \epsilon}{8 \pi} E_{o}^{2}=\cos \theta \frac{v_{g} \epsilon}{8 \pi} E_{o}^{\prime 2}+\cos \theta^{\prime} \frac{v_{g}^{\prime} \epsilon^{\prime}}{8 \pi} E_{o}^{\prime \prime 2}
$$

Substituting $E_{o}^{\prime}=r E_{o}, E_{o}^{\prime \prime}=t E_{o}$ and dividing both sides by the incoming flux yields

$$
1=R+T=|r|^{2}+|t|^{2} \frac{v_{g}^{\prime}}{v_{g}} \frac{\epsilon^{\prime}}{\epsilon} .
$$

The reflectance is identified by $R=|r|^{2}$ and transmittance by $T=1-R=|t|^{2}\left(v_{g}^{\prime} / v_{g}\right)\left(\epsilon^{\prime} / \epsilon\right)$. Notice that $R=|r|^{2}$ but $T=1$ $-|r|^{2} \neq|t|^{2}$ due to the difference in power density and propagation angle after the interface. It is thus easier to use reflectance $|r|^{2}$ for both transmission and reflection.

\section{APPENDIX B: PARAXIAL PROPAGATION}

We seek the free-space evolution for a Cosine-GaussHermite $U\left(x_{o} ; 0\right)=e^{ \pm k_{m} x_{o}} H_{n}\left(\sqrt{2} x_{o} / w_{o}\right) \exp \left[-x_{o}^{2} / w_{o}^{2}\right]$, given by the one-dimensional paraxial propagator

$$
U(x ; z)=\int d x_{o} U\left(x_{o} ; 0\right) \sqrt{\frac{i k}{2 \pi z}} \exp \left[i k_{o} \frac{\left(x-x_{o}\right)^{2}}{2 z}\right] .
$$

Consider the expression

$$
\int_{-\infty}^{\infty} d \chi_{o} \exp \left[-s^{2}+2 s \chi_{o}-\frac{\chi_{o}^{2}}{2}+\frac{\alpha}{2}\left(\chi-\chi_{o}\right)^{2}-2 \beta \chi_{o}\right]
$$

with the notation $\alpha=i b / z, \quad \chi=x \sqrt{2} / w_{o}$, and $\beta=i k_{x} w_{o} / 2 \sqrt{2}$. Completing the squares in the exponent recasts Eq. (B2) as

$$
\begin{aligned}
& \exp \left[-\bar{s}^{2}+2 \bar{s} \bar{\chi}-\frac{\bar{\chi}^{2}}{2}\right] \exp \left[-\frac{1}{2} \frac{\alpha}{\alpha^{2}-1} \chi^{2}-\frac{2 \alpha}{\alpha^{2}-1} \beta^{2}\right. \\
& \left.+\frac{4 \alpha^{2}}{\alpha^{2}-1} \beta \chi\right] \\
& \times \int_{-\infty}^{\infty} d \chi_{o} \exp \left[-\frac{1-\alpha}{2} \chi_{o}^{2}+2\left(s+\beta-\frac{\alpha}{2}\right) \chi_{o}\right. \\
& \left.-\frac{2}{1-\alpha}\left(s+\beta-\frac{\alpha}{2}\right)^{2}\right]
\end{aligned}
$$

where $\hat{s}=s \sqrt{(1+\alpha) /(1-\alpha)}$ and $\hat{\chi}=\alpha / \sqrt{\alpha^{2}-1}(\chi-\beta / 2 \alpha)$. The right-hand integral yields just $2 \pi / \sqrt{1-\alpha}$. Now, using generating function definition for Gauss-Hermite, the terms exp[ $\left.-s^{2}+2 s \chi-\left(\chi^{2} / 2\right)\right]$ appearing on both Eqs. (B2) and (B3) are expanded in powers of $s^{m}$ as

$$
\exp \left[-s^{2}+2 s \chi-\frac{\chi^{2}}{2}\right]=\sum_{m=0}^{\infty} \frac{s^{m}}{m !} H_{m}(\chi) e^{-\chi^{2} / 2},
$$

the Gauss-Hermite functions $\psi_{m}(\chi)=H_{m}(\chi) \exp \left[-\chi^{2} / 2\right]$ being the expansion coefficients. After the expansion and a term-by-term equation among the same powers $s^{m}$ on both sides of Eqs. (B2) and (B3) yield

$$
\begin{aligned}
& \int_{-\infty}^{\infty} d \chi_{o} \exp \left[\frac{\alpha}{2}\left(\chi-\chi_{o}\right)^{2}\right] \psi_{m}\left(\chi_{o}\right) \exp \left[2 \beta \chi_{o}\right] \\
& =\sqrt{\frac{2 \pi}{1-\alpha}}\left(\frac{1+\alpha}{1-\alpha}\right)^{m / 2} H_{m}(\hat{\chi}) \exp \left[-\frac{1}{2} \hat{\chi}^{2}(1+\alpha)\right] \\
& \quad \times \exp \left[-\alpha \frac{\chi^{2}}{2}\right]
\end{aligned}
$$

Multiplying both sides by the remaining propagator factor $\sqrt{i k_{o} / 2 \pi z}$ in Eq. (B1), and by $w_{o} / \sqrt{2}$ to convert to $x$ integration, after symbol manipulations $\left(\alpha=i b / z=i k_{o} w_{o}^{2} / 2 z\right)$ the left hand equals $u_{m n}(x ; z)$, the convolution of the Cosine-GaussHermite with the paraxial propagator

$$
\begin{aligned}
u_{m n}(x ; z)= & \int_{-\infty}^{\infty} d x_{o} \sqrt{\frac{i k}{2 \pi z}} \exp \left[i k \frac{\left(x-x_{o}\right)^{2}}{2 z}\right] \psi_{m} \\
& \times\left(\sqrt{2} x / w_{o}\right) \exp \left[i k_{n} x_{o}\right] .
\end{aligned}
$$

By the same token, the right-hand side of Eq. (B5) multiplied by $\sqrt{i k_{o} w_{0}^{2} / 4 \pi z}=\sqrt{i b / 2 \pi z}$, using $\sqrt{i b / 2 \pi z} \sqrt{2 \pi /(1-\alpha)}$ $=e^{-i \varphi(z) / 2} / \sqrt{1+z^{2} / b^{2}},[(1+\alpha) /(1-\alpha)]^{m / 2}=e^{-i m \varphi(z)}$, and converting $\hat{\chi}=\alpha / \sqrt{\alpha^{2}-1}[\chi-(\beta / 2 \alpha)]=\sqrt{2}\left(x-z \sin \theta_{n}\right) / W(z)$, yields the sought after the rhs of Eq. (15). The derivation is completed by multiplying both sides by the normalization factor $C_{m n}\left(2 / \pi w_{o}^{2}\right)^{1 / 4}$. Because paraxial propagation conserves flux, the normalization constant is obtained at the waist $z=0$. It can be shown that $C_{m 0}=1 / \sqrt{2^{m} m !}$ and $C_{0 n}$ $=1 / \sqrt{2\left(1+\exp \left[-k_{n}^{2} w_{o}^{2} / 2\right]\right)}$.

\section{APPENDIX C: REFLECTION COUPLING COEFFICIENT}

We assume that a collimating mirror with matched radius of curvature and focal distance is focusing the radiation in the fast diffracting $y$ direction and minimizing the $y$-diffraction effects. Thus, the overlap integral (8), $V_{i, i \pm 1}$ $=V_{12}$, is approximated by

$$
\begin{aligned}
V_{12} & =\int d \mathbf{r}^{2} U(\mathbf{r} ; 0) U(\mathbf{r}+d ; z=2 l) \\
& =\int d y \int d x s(y ; 0) s(y ; 2 l) u_{m n}(x ; 0) u_{m n}(x+d ; 2 l) \\
& \simeq \int d y s^{2}(y) \int d x u_{m n}(x ; 0) u_{m n}(x+d ; 2 l) \\
& =\int d x u_{m n}^{ \pm}(x ; 0) u_{m n}(x+d ; 2 l)
\end{aligned}
$$

because of $s(y ; z=2 l) \simeq s(y ; z=0)$ and the implicit normaliza- 
tion $\int d y s^{2}(y)=1$. Diffraction enters from the free diffraction along the "slow" $x$ axis. In Eq. (C1), the target cavity profile $u_{m n}^{ \pm}(x ; 0)$ and the diffracted feedback radiation profile $u_{m n}^{ \pm}(x$ $+d ; z=2 l)$ are given, respectively, by Eqs. (14) and (15). We limit the overlap calculation for the Cosine-Gaussian mode $m=0$. Taking into account the separation $d$ between the two cavity centers and expressing the diffracted beam coordinates $x^{\prime}$ in target cavity coordinates $x^{\prime}=x+d=x+X_{n}+\delta$, where $X_{n} \equiv 2 l \sin \theta_{n}$ is the center location for the reflected wavefront and $\delta=d-X_{n}$ is its offset from the target cavity center, yields

$$
\begin{aligned}
V_{0 n}^{++}= & C_{0 n}^{2}\left[\frac{4}{\pi^{2} w_{o}^{2} W^{2}(2 l)}\right]^{1 / 4} \int d x\left[e^{+i k_{n} x} e^{-x^{2} / w_{o}^{2}}\right]^{*} \\
& \times e^{-(i / 2) \varphi(2 l)} e^{-[1+i(2 l / b)]\left[\left(x-x_{n}\right)^{2} / W^{2}(2 l)\right]} \\
& \times e^{-i k_{o}\left[\left(x+X_{n}+\delta\right)^{2} / 2(2 l)\right]},
\end{aligned}
$$

with $W^{2}(2 l)=w_{o}^{2}\left[1+(2 l / b)^{2}\right]$. The flip in the wavefront curvature from the reflection at the grating has been included in Eq. (C2). After rearranging terms the $x$ integral becomes

$$
\begin{aligned}
I_{++}= & e^{i k_{o}\left[\left(X_{n}+\delta\right)^{2} / 2(2 l)\right]} \int d x e^{-\left(1 / w_{o}^{2}\right)\left[x^{2}(1+\xi)+2 x \xi \delta+\delta^{2} \xi\right]} \\
= & e^{-i k_{o}\left[\left(X_{n}+\delta\right)^{2} / 2(2 l)\right]} e^{-\left(\delta^{2} / w_{o}^{2}\right) \xi} \\
& \times \int d x e^{-\left[(1+\xi) / w_{o}^{2}\right]\left[x^{2}+2(\xi \delta /(1+\xi)) x\right]},
\end{aligned}
$$

where $\xi \equiv 1 /[1-i(2 l) / b]=[1+i(2 l) / b] /\left[1+(2 l)^{2} / b^{2}\right] . \quad$ Expressing the integral in terms of the error function $\operatorname{erf}(y)$ $=(1 / \sqrt{\pi}) \int_{0}^{\infty} d x e^{-x^{2}}$ and putting back in Eq. (C2) yields

$$
\begin{aligned}
V_{0 n}^{++}= & C_{0 n}^{2}\left[\frac{w_{o}^{2}}{W^{2}(2 l)}\right]^{1 / 4} \sqrt{\frac{2}{1+\xi}} e^{-i[\varphi(2 l) / 2]} \\
& \times e^{\left.i\left[\left[k_{o} X_{n}\left(X_{n}+2 \delta\right)\right]\right][2(2 l)]\right\}} e^{-\left(\delta^{2} / w_{o}^{2}\right)[\xi(\xi /(1+\xi)]} \\
& \frac{1}{2}\left[\operatorname{erf}\left(\frac{a \sqrt{1+\xi}}{w_{o}}+\frac{\xi \delta}{w_{o} \sqrt{1+\xi}}\right)\right. \\
& \left.-\operatorname{erf}\left(-\frac{a \sqrt{1+\xi}}{w_{o}}+\frac{\xi \delta}{w_{o} \sqrt{1+\xi}}\right)\right] .
\end{aligned}
$$

The calculation of $V_{0 n}^{-}$involves the change of signs $x_{n} \rightarrow$ $-x_{n}, \delta \rightarrow-\delta$ and yields the same result, Eq. (C2). Note that $V^{++}\left(V^{--}\right)$expresses the coupling between same transverse wave components $e^{i k_{n} x}\left(e^{-i k_{n} x}\right)$ among two cavities. In the beating between the reflected and the target cavity fields the fast phase $\propto \exp \left[i 2 k_{o} x x_{n} / 4 l\right]$ inside the far-field curvature $\exp \left[i 2 k_{o}\left(x+X_{n}\right)^{2} / 2(2 l)\right]$ cancels out the fast transverse phase $\left(e^{i k_{n} x}\right)^{*}=e^{-i k_{n} x}$ in the target cavity near field, $i k_{o} x\left(X_{n} / 2 l\right.$ $\left.-k_{n} / k_{o}\right)=0$, since $x_{n} / 2 l=\sin \theta_{n}=k_{n} / k_{o}$. On the other hand, the coupling coefficient between opposite transverse wavenumbers

$$
\begin{aligned}
V_{0 n}^{+-}= & V_{0 n}^{+*}=C_{m}^{2}\left[\frac{4}{\pi^{2} w_{o}^{2} W^{2}(2 l)}\right]^{1 / 4} \int d x\left[e^{-i k_{n} x} e^{-x^{2} / w_{o}^{2}}\right]^{*} \\
& \times e^{-(i / 2) \varphi(2 l)} \\
& \times e^{-[1+i(2 l / b)]\left[\left(x-X_{n}\right)^{2} / W^{2}(2 l)\right]} \\
& \times e^{-i k_{o}\left[\left(x+X_{n}+\delta\right)^{2} / 2(2 l)\right]}
\end{aligned}
$$

contains a fast varying phase $\exp \left[i k_{o} x\left(X_{n} / 2 l+k_{n} / k_{o}\right)\right]$ $=\exp \left[2 i k_{o} \sin \theta_{n} x\right]=\exp \left[4 i\left(b / w_{o}^{2}\right) \sin \theta_{n} x\right]$, producing the overlap integral

$$
\begin{aligned}
I_{+-}= & e^{i k_{o}\left[\left(X_{n}+\delta\right)^{2} / 2(2 l)\right]} \int d x e^{-\left(1 / w_{o}^{2}\right)\left[x^{2}(1+\xi)+2 x\left(\xi \delta+2 i b \sin \theta_{n}\right)+\delta^{2} \xi\right]} \\
= & e^{-i k_{0}\left[\left(X_{n}+\delta\right)^{2} / 2(2 l)\right]} e^{-\left(\delta^{2} / w_{o}^{2}\right) \xi} \\
& \times \int d x e^{-\left[(1+\xi) / w_{o}^{2}\right]\left\{x^{2}+2\left[\left(\xi \delta+2 i b \sin \theta_{n}\right) /(1+\xi)\right] x\right\}}
\end{aligned}
$$

instead of Eq. (C3). Integration and substitution in Eq. (C5) yields or the fundamental Cosine-Gaussian $m=0$,

$$
\begin{aligned}
V_{0 n}^{ \pm \mp}= & C_{0 n}^{2}\left[\frac{w_{o}^{2}}{W^{2}(2 l)}\right]^{1 / 4} \sqrt{\frac{2}{1+\xi}} e^{-i[\varphi(2 l) / 2]} \\
& \times e^{i\left\{\left[k_{o} X_{n}\left(X_{n}+2 \delta\right)\right] / 2(2 l)\right\}} e^{i\left\{\left\langle\xi \delta b \sin \theta_{n}\left[w_{o}^{2}(1+\xi)\right]\right\}\right.} \\
& \times e^{-\left(\delta^{2} \xi+4 b^{2} \sin ^{2} \theta_{n}\right) /\left[w_{o}^{2}(1+\xi)\right]} \\
& \frac{1}{2}\left[\operatorname{erf}\left(\frac{a \sqrt{1+\xi}}{w_{o}}+\frac{\xi \delta+2 i b \sin \theta_{n}}{w_{o} \sqrt{1+\xi}}\right)\right. \\
& \left.-\operatorname{erf}\left(-\frac{a \sqrt{1+\xi}}{w_{o}}+\frac{\xi \delta+2 i b \sin \theta_{n}}{w_{o} \sqrt{1+\xi}}\right)\right]
\end{aligned}
$$

Combining Eqs. (C4) and (C7) yields

$$
\begin{aligned}
V_{12}^{0 n}= & \frac{1}{2\left(1+\exp \left[-k_{n}^{2} w_{o}^{2} / 2\right]\right)}\left[\frac{w_{o}^{2}}{W^{2}(2 l)}\right]^{1 / 4} \sqrt{\frac{2}{1+\xi}} \\
& \times e^{-i[\varphi(2 l) / 2]} e^{i\left[\left[k_{o} X_{n}\left(X_{n}+2 \delta\right)\right] / 2(2 l)\right\}} \\
& \times e^{-\left(\delta^{2} / w_{o}^{2}\right)[\xi \xi(1+\xi)]}\left\{\frac { 1 } { 2 } \left[\operatorname{erf}\left(\frac{a \sqrt{1+\xi}}{w_{o}}+\frac{\xi \delta}{w_{o} \sqrt{1+\xi}}\right)\right.\right. \\
& \left.-\operatorname{erf}\left(-\frac{a \sqrt{1+\xi}}{w_{o}}+\frac{\xi \delta}{w_{o} \sqrt{1+\xi}}\right)\right] \\
& +e^{-4 b^{2} \sin ^{2} \theta_{n} /\left[w_{o}^{2}(1+\xi)\right]} e^{i\left\langle 4 \xi \delta b \sin \theta_{n} /\left[w_{o}^{2}(1+\xi)\right]\right\}} \\
& \frac{1}{2}\left[\operatorname{erf}\left(\frac{a \sqrt{1+\xi}}{w_{o}}+\frac{\xi \delta+2 i b \sin \theta_{n}}{w_{o} \sqrt{1+\xi}}\right)\right. \\
& \left.\left.-\operatorname{erf}\left(-\frac{a \sqrt{1+\xi}}{w_{o}}+\frac{\xi \delta+2 i b \sin \theta_{n}}{w_{o} \sqrt{1+\xi}}\right)\right]\right\} .
\end{aligned}
$$

This is the full paraxial result for sidelobe overlap. For the usual parameter scaling $b^{2} / w_{o}^{2}=k_{o}^{2} w_{o}^{2} / 4 \geq k_{n}^{2} w_{o}^{2} \gg 1$, the second summand inside the curly bracket, representing $V^{+-}$for the coupling among opposite transverse wavenumbers $\pm k_{n}$, is negligible. When in addition the cavity width is larger than the mode waist $a / w_{o}>2$ the erf square bracket tends to 
unity, and Eq. (C8) with substitutions for $\xi$, yields Eq. (17).

\section{APPENDIX D: REFLECTION BY BRAGG GRATING: BEAM EXPANSION AND EFFECTIVE GRATING DISTANCE}

The long interaction path inside the thick, low-index contrast Bragg grating, employed for sidelobe reflection, causes significant changes in the reflected wavefront. Due to the distributed interaction, the total reflected radiation at the leading grating edge $z=l$ is a superposition of partial wavefronts $d E(z)$ reflected from different depths $\bar{z} \equiv z-l$ within the grating thickness $G$. Due to different traveled distances $2 \bar{z}$, wavefronts reflected off deeper layers yield increasingly expanded (diffracted) widths $W(l+\bar{z})$ with laterally displaced centers by $x=2 \bar{z} \sin \theta^{\prime}$, where the diffracted angle $\sin \theta^{\prime}$ $=\sin \theta / n$. Applying the paraxial propagation to each partial wavefront, and summing up over $\bar{z}$, the reflected wavefront at $\bar{z}=0(z=l)$ for the incoming Gaussian $E(x, y ; l)$ $=E_{o} \sqrt{k b / \pi}[1 /(l+i b)] e^{i k\left[\left(x^{2}+y^{2}\right) /(2 l+i b)\right]}$ is given by

$$
\begin{aligned}
E^{\prime}(x, y ; l)= & \int_{0}^{G} d E(\bar{z}) \sqrt{\frac{n k n b}{\pi}} \frac{1}{(n l+\bar{z})+i n b} \\
& \times \exp \left\{i n k \frac{\left(x-2 \bar{z} \sin \theta_{x}^{\prime}\right)^{2}+\left(y-2 \bar{z} \sin \theta_{y}^{\prime}\right)^{2}}{2[(n l+\bar{z})+i n b]}\right\} .
\end{aligned}
$$

Note that propagation inside a grating of average refraction index $n$ involves wavefronts stemming from an "image" waist $w_{o}$ at distance $n l$, where $l$ is the actual free-space distance of the incoming beam waist $w_{o}$ from the grating leading edge. The diffraction length is $n b$, where $b=k w_{o}^{2} / 2$, the free-space Rayleigh length. Now, for small index contrast, the power distribution inside the grating is given to a very good approximation by the exponential $E^{2}(\bar{z})=E_{o}^{2} e^{-2 \kappa \bar{z}}$, where $\kappa$ is related to the reflectance $R$ by $\kappa=-\ln R / 2 G$, thus the partially reflected amplitude from $\bar{z}$ is $d E(\bar{z})=d \bar{z} \kappa E_{o} e^{-\kappa \bar{z}}$. Substitution in Eq. (D1) yields Eq. (18). Equation (18) is exact within the paraxial framework.

The lateral shift in the reflected wavefront center can be found from the location $x_{o}$ of the peak $\partial \Re E^{\prime}(x, y ; l) /\left.\partial x\right|_{x_{o}}$ $=0$. Using Eq. (18) yields an integral equation for $\Delta_{x} \equiv x_{o}$,

$$
\begin{gathered}
\int_{0}^{G} d \bar{z} \kappa \exp [-\kappa \bar{z}] \frac{22\left(\Delta_{x}-2 \bar{z} \sin \theta_{x} / n\right)}{\pi w_{o}^{2}\left[1+(n l+\bar{z})^{2} /(n b)^{2}\right]^{2}} \\
\quad \times \exp \left\{-\frac{\left(\Delta_{x}-2 \bar{z} \sin \theta_{x} / n\right)^{2}}{w_{o}^{2}\left[1+(n l+\bar{z})^{2} /(n b)^{2}\right]}\right\}=0 .
\end{gathered}
$$

The penetration depth $\delta l_{x}^{\prime}$ is defined by $\Delta_{x}=2 \sin \theta_{x}^{\prime} \delta l_{x}^{\prime}$, as if all radiation has been reflected from a layer at depth $\delta l_{x}^{\prime}$ inside the grating. The emerging from the grating wavefronts appear as reflected from a free-space penetration depth $\delta l_{x}$ $=\delta l^{\prime} / n$, Fig. 4(c), with $\Delta_{x}=2 \sin \theta_{x} \delta l_{x}$

Analytic estimates of the waist expansion are possible by taking the mean value of the radiation spot size around the shifted axis $\left\langle\left(x-\Delta_{x}\right)^{2}+\left(y-\Delta_{y}\right)^{2}\right\rangle$ over power. For Gaussian beams the $1 / e$-width equals twice the $\operatorname{rms}$ spot size $\left\langle W_{x}^{2}\right\rangle$
$=2\left\langle\left(x-\Delta_{x}\right)^{2}\right\rangle$, etc. Using the absolute square of the radiation amplitude inside Eq. (18), the fact that $d P(z)=-(2 \kappa) P(z)$, and weighting by the total reflected power $R$ $=\int_{0}^{G} d \bar{z}(2 \kappa) e^{-2 \kappa \bar{z}}=1-\exp [-2 \kappa G]$, one has

$$
\begin{aligned}
\left\langle W_{x}^{2}\right\rangle= & \frac{2}{R} \int_{0}^{G} d \bar{z} 2 \kappa \exp [-2 \kappa \bar{z}] \\
& \times \frac{2}{\pi w_{o}^{2}\left[1+(n l+\bar{z})^{2} /(n b)^{2}\right]} \\
& \times \int_{-\infty}^{\infty} \int_{-\infty}^{\infty} d x d y\left(x-\Delta_{x}\right)^{2} \\
& \times \exp \left\{-\frac{\left(x-2 \bar{z} \sin \theta_{x} / n\right)^{2}+\left(y-2 \bar{z} \sin \theta_{y} / n\right)^{2}}{w_{o}^{2}\left[1+(n l+\bar{z})^{2} /(n b)^{2}\right]}\right\},
\end{aligned}
$$

where use was made of the definition $b=k w_{o}^{2} / 2$. Substituting $\Delta_{x, y}=2 \delta l_{x, y} \sin \theta_{x, y}$, changing variables to $\bar{x}=x-2 \bar{z} \sin \theta_{y} / n$, $\bar{y}-2 \bar{z} \sin \theta_{y} / n$, the double integral becomes

$$
\begin{aligned}
& \int_{-\infty}^{\infty} \int_{-\infty}^{\infty} d \bar{x} d \bar{y}\left[\bar{x}+2 \sin \theta_{x}\left(\frac{\bar{z}}{n}-\delta_{x}\right)\right]^{2} \\
& \times \exp \left\{-\frac{\bar{x}^{2}+\bar{y}^{2}}{w_{o}^{2}\left[1+(n l+\bar{z})^{2} /(n b)^{2}\right]}\right\} .
\end{aligned}
$$

Performing the $y$ integration, and since odd powers of $\bar{x}$ yield null during $x$ integration

$$
\begin{aligned}
& \sqrt{\frac{\pi w_{o}^{2}\left[1+(n l+\bar{z})^{2} /(n b)^{2}\right]}{2}} \int_{-\infty}^{\infty} d \bar{x}\left[\bar{x}^{2}+4 \sin ^{2} \theta_{x}\right. \\
& \left.\quad \times\left(\frac{\bar{z}}{n}-\delta_{x}\right)^{2}\right] \exp \left\{-\frac{\bar{x}^{2}}{w_{o}^{2}\left[1+(n l+\bar{z})^{2} /(n b)^{2}\right]}\right\} \\
& =\frac{\pi w_{o}^{2}\left[1+(n l+\bar{z})^{2} /(n b)^{2}\right]}{2}\left\{\frac{w_{o}^{2}\left[1+(n l+\bar{z})^{2} /(n b)^{2}\right]}{2}\right. \\
& \left.+4 \sin ^{2} \theta_{x}\left(\frac{\bar{z}}{n}-\delta_{x}\right)^{2}\right\} .
\end{aligned}
$$

Substituting inside Eq. (D3) yields Eq. (19), and similar for $W_{y}$. Analytic evaluation is possible with the approximation $\delta l_{x} \simeq 1 / 2 \kappa$

We digress to suggest that a more accurate treatment of the reflected radiation involves an expansion of the reflected wavefront (D2) into a superposition of Gauss-Hermite (G-H) modes, where the expansion coefficients are determined by the integral projections into the corresponding $\mathrm{G}-\mathrm{H}$ mode. This has the advantage that further propagation of the reflected radiation is given in exact form for each $\mathrm{G}-\mathrm{H}$ mode by the usual waist and curvature paraxial transformation. Many equivalent representations exist, depending on the choice of the reflected mode waist for the G-H family. The optimum representation corresponds to finding the reflected waist value $W^{\prime}$ that maximizes the reflection coefficient into the fundamental mode. A variational principle has been formulated for that purpose. 
ACKNOWLEDGMENT

This work is supported by DARPA Contract No. HR0011-06-1-0010.

${ }^{1}$ J. K. Butler, D. E. Ackley, and M. Ettenberg, IEEE J. Quantum Electron. 21, 458 (1985).

${ }^{2}$ J.-M. Verdiell and R. Frey, IEEE J. Quantum Electron. 26, 270 (1990).

${ }^{3}$ R. M. R. Pillai and E. M. Garmire, IEEE J. Quantum Electron. 32, 996
(1996).

${ }^{4}$ D. Botez and A. P. Napartovich, IEEE J. Quantum Electron. 30, 975 (1994); 32, 2175 (1996).

${ }^{5}$ G. Hergenhan, B. Lucke, and U. Brauch, Appl. Opt. 42, 1667 (2003).

${ }^{6}$ G. B. Venus, A. Sevian, V. I. Smirnov, and L. B. Glebov, Opt. Lett. 31, 1453 (2006)

${ }^{7}$ S. Riyopoulos, Phys. Rev. A 66, 053820 (2002).

${ }^{8}$ S. Riyopoulos, J. Opt. Soc. Am. B 23, 250 (2006).

${ }^{9}$ S. Riyopoulos, IEEE J. Quantum Electron. 41, 1450 (2005). 